OPEN ACCESS

Edited by:

lolanda Leite,

Royal Institute of Technology, Sweden

Reviewed by:

Anouk Van Maris,

Bristol Robotics Laboratory,

United Kingdom

Álvaro Castro González,

Universidad Carlos III de Madrid

Spain

*Correspondence:

Marcela Múnera

marcela.munera@escuelaing.edu.co

Received: 25 November 2020 Accepted: 17 February 2021

Published: 16 March 2021

Citation:

Céspedes N, Irfan B, Senft $E$,

Cifuentes CA, Gutierrez LF,

Rincon-Roncancio M, Belpaeme T and Múnera M (2021) A Socially

Assistive Robot for Long-Term

Cardiac Rehabilitation in the Real

World. Front. Neurorobot. 15:633248.

doi: $10.3389 /$ fnbot.2021.633248

\section{A Socially Assistive Robot for Long-Term Cardiac Rehabilitation in the Real World}

\author{
Nathalia Céspedes ${ }^{1}$, Bahar Irfan ${ }^{2}$, Emmanuel Senft ${ }^{2,3}$, Carlos A. Cifuentes ${ }^{1}$, \\ Luisa F. Gutierrez ${ }^{4}$, Mónica Rincon-Roncancio ${ }^{4}$, Tony Belpaeme ${ }^{2,5}$ and Marcela Múnera ${ }^{1 *}$ \\ ${ }^{1}$ Biomedical Engineering Department, Colombian School of Engineering Julio Garavito, Bogotá, Colombia, ${ }^{2}$ Centre for \\ Robotics and Neural Systems, University of Plymouth, Plymouth, United Kingdom, ${ }^{3}$ Department of Computer Sciences, \\ University of Wisconsin-Madison, Madison, WI, United States, ${ }^{4}$ Fundación Cardioinfantil-Instituto de Cardiología, Bogotá, \\ Colombia, ${ }^{5}$ IDLab-imec, Ghent University, Ghent, Belgium
}

What are the benefits of using a socially assistive robot for long-term cardiac rehabilitation? To answer this question we designed and conducted a real-world long-term study, in collaboration with medical specialists, at the Fundación Cardioinfantil-Instituto de Cardiología clinic (Bogotá, Colombia) lasting 2.5 years. The study took place within the context of the outpatient phase of patients' cardiac rehabilitation programme and aimed to compare the patients' progress and adherence in the conventional cardiac rehabilitation programme (control condition) against rehabilitation supported by a fully autonomous socially assistive robot which continuously monitored the patients during exercise to provide immediate feedback and motivation based on sensory measures (robot condition). The explicit aim of the social robot is to improve patient motivation and increase adherence to the programme to ensure a complete recovery. We recruited 15 patients per condition. The cardiac rehabilitation programme was designed to last 36 sessions (18 weeks) per patient. The findings suggest that robot increases adherence (by 13.3\%) and leads to faster completion of the programme. In addition, the patients assisted by the robot had more rapid improvement in their recovery heart rate, better physical activity performance and a higher improvement in cardiovascular functioning, which indicate a successful cardiac rehabilitation programme performance. Moreover, the medical staff and the patients acknowledged that the robot improved the patient motivation and adherence to the programme, supporting its potential in addressing the major challenges in rehabilitation programmes.

Keywords: social assistive robotics, cardiac rehabilitation, human-robot interaction, long-term interaction, social robot, human-robot interface

\section{INTRODUCTION}

Cardiovascular diseases (CVDs) are a group of disorders of the heart and blood vessels that include cerebrovascular diseases, rheumatic heart diseases and other conditions (World Health Organization, 2011). CVDs are the cause of 17.7 million deaths every year, approximately $31 \%$ of all deaths worldwide (World Health Organization, 2011). Within the CVDs, ischemic heart disease 
causes 8.76 million deaths, and strokes cause 6.24 million deaths ${ }^{1}$ worldwide each year.

Cardiac rehabilitation (CR) following a cardiovascular event is a Class I recommendation of the European Society of Cardiology, the American Heart Association, and the American College of Cardiology (Thomas et al., 2007; Piepoli et al., 2010; Galve et al., 2014). A typical CR programme promotes a healthy lifestyle, reduces risk factors, improves health-related quality of life, and decreases mortality and morbidity (Oldridge et al., 1988; Taylor et al., 2004, 2012; Clark et al., 2005; Kraus and Keteyian, 2007; Lawler et al., 2011; Anderson et al., 2016; Giuliano et al., 2017). CR programme is generally conducted in three phases (Kraus and Keteyian, 2007): (I) inpatient, (II) outpatient, and (III) maintenance phase. The inpatient phase starts after the patient is hemodynamically stable, typically after $48 \mathrm{~h}$ of the surgery procedure. The patient performs low-intensity movements for maintaining muscle tone and reducing risks or any complication at this phase. The outpatient phase starts after the patient is discharged, and lasts on average 18 weeks with sessions twice per week. During this phase, the patient performs physical exercises at the hospital, and receives an educational programme about the risk factors to gain healthy habits (e.g., controlling blood pressure, cholesterol, weight, and stress management). The maintenance phase aims to reinforce the information and habits gained during the outpatient phase and lasts on average about 9 months with one or two sessions per week. The physical exercises in the outpatient phase typically last $1 \mathrm{~h}$, and consist of (1) warmup via stretching exercises, (2) training by physical exercises, e.g., on a treadmill, and (3) cooldown during which low-intensity exercises are carried out. In a conventional CR session, during warm-up and cooldown, the medical staff measures the initial and final heart rate and blood pressure. During training, the heart rate and the exertion level of the patient are requested by the medical staff regularly to determine whether there is a need for the intervention to decrease the intensity of the exercise, which is determined by the speed and inclination of the treadmill. Our work focuses on the training step to provide individual and immediate feedback during the workout, and alert the medical staff in the case of critical biomedical values.

Adherence to the CR programme is vital for the complete recovery of a patient and to reduce the risk of suffering recurrent events (Jolly et al., 2007; Suaya et al., 2009; Hammill et al., 2010). Nonetheless, in addition to the low participation in the programme (Altenhoener et al., 2005; McKee et al., 2014), a high percentage (24-50\%) of patients who enroll in cardiac rehabilitation programmes drop out (Carlson et al., 2000; Scane et al., 2012). The reasons behind dropout or nonattendance vary, such as motivation factors, lack of interest or faith in the CR programme, increasing lack of motivation throughout the CR programme (Siegert and Taylor, 2004; McKee et al., 2014), anxiety about the exercise component (Cooper et al., 2007), a lack of group cohesion (Maclean and Pound, 2000; Beswick et al., 2005), presence of comorbidities (e.g., depression, obesity, diabetes) (Turk-Adawi et al., 2013), poor

\footnotetext{
${ }^{1}$ World Health Organization on cardiovascular diseases: https://www.who.int/ health-topics/cardiovascular-diseases.
}

funding or poor organization (Bethell et al., 2009), difficulties with the location or accessibility (Turk-Adawi et al., 2013), scheduling or work commitments (Ruano-Ravina et al., 2016), and negative beliefs (Shahsavari et al., 2012). While other factors are beyond the control of the healthcare staff, motivational issues can be addressed by providing individual support within the sessions, through rigorous supervision during the patient's exercise and quick help in emergent situations (Shahsavari et al., 2012). However, the CR programme at clinics is generally conducted with large groups, and it is challenging for healthcare staff to provide continuous and individual support during the session (Turk-Adawi et al., 2019). In this context, integrating a socially assistive robot (Feil-Seifer and Matarić, 2005) can help provide one-on-one support to the patient, which, in turn, can facilitate the healthcare staff to focus on the individual needs of patients, immediately detect any complications during the session, analyse the patient's progress within the programme in more detail and provide a more tailored plan.

Based on this, this paper presents a real-world long-term study where a socially assistive robot was used to provide motivation and feedback to the patients, aimed to support CR phase II therapies and improve the adherence. This is the first in-depth clinical study that explores the benefits of using a socially assistive robot for long-term cardiac rehabilitation in terms of adherence and physiological progress. Furthermore, in contrast to our previous studies, where we analyzed patients on a case-by-case basis, this paper includes the analysis for the physiological progress through the complete CR programme (36 sessions) for all the patients recruited during the study, in addition to the perceptions of the clinicians that were part of the study for 2.5 years. The remainder of this work is organized as follows. section 2 presents Socially Assistive Robotics (SAR) studies focused on healthcare conditions. section 3 describes the interface architecture used in the CR environment. section 4 presents the protocol and experimental design carried out. section 5 presents the results from the study, section 6 highlights findings and discusses the implications, and section 7 presents the conclusions of the study.

\section{RELATED WORK}

Socially Assistive Robotics (SAR) is a domain of Human-Robot Interaction (HRI) which focuses on developing robots capable of assisting users through social interaction (Feil-Seifer and Matarić, 2005; Matarić and Scassellati, 2016). Unlike virtual agents, socially assistive robots present a physical embodiment, which improves likeability (Fasola and Matarić, 2013; Li, 2015), user engagement and motivation (Vasco et al., 2019), adherence (Bickmore and Picard, 2005; Kidd and Breazeal, 2007) and task performance (Vasco et al., 2019), which are essential in long-term healthcare programmes.

Socially assistive robots have been shown to improve user motivation and engagement in several studies in rehabilitation (Kang et al., 2005; Gockley and Mataric, 2006; Matarić et al., 2007a; Fasola and Matarić, 2010; Fasola and Mataric, 2012; Šabanović et al., 2013; Swift-Spong et al., 2015), 
in addition to improved adherence (Gadde et al., 2011). Moreover, for repetitive exercise during healthcare programmes, encouraging feedback and continuous monitoring are essential to enhance motivation and task performance (Eriksson et al., 2005; Kang et al., 2005). Most research in rehabilitation has been carried out under laboratory conditions or during short-term interventions, which restrict the applicability of their impact in real-world clinical scenarios for long-term rehabilitation, due to the confounding factors, such as the novelty effect (Gockley et al., 2005) and the adaptation of the technology (Leite et al., 2013; Lane et al., 2016; Riek, 2017).

The only prior study in the literature that evaluates using a socially assistive robot in cardiac rehabilitation is that of Kang et al. (2005). A hands-off physical therapy assistant robot was used in spirometry exercises, which the patients were satisfied with. However, the study only analyzed short-term benefits (one session), with a low number of (5) healthy participants under laboratory conditions, and it did not analyse the physiological progress of the patients. Socially assistive robots were also explored within other similar areas of rehabilitation, such as post-stroke rehabilitation, which showed potential benefits, such as increased willingness to perform prescribed exercises and enthusiastic responses toward the robot (Matarić et al., 2007a).

Perceptions of healthcare staff toward using SAR may be initially negative due to the common concerns or doubts (Winkle et al., 2018; Casas et al., 2019). A common negative perception toward robots is believing them to be potential replacements of their job. However, SAR relies on the collaboration of the medical staff to provide individual support to the patient and help the medical staff in monitoring the patients more closely. Some studies have shown that these perceptions can improve after demonstrating the benefits of using a socially assistive robot (Winkle et al., 2018; Casas et al., 2019). In addition, clinicians' trust may increase with the repeated use of the robot and by positive recommendations of other healthcare professionals in the field (Carrillo et al., 2018). The adoption of the technology by the healthcare staff depends heavily on the system's reliability and their resulting trust (Winkle et al., 2018; Langer et al., 2019). Nonetheless, incorporating social robots into the real-world healthcare programmes poses several challenges, such as technical failures of the robot or sensors, which may negatively affect the perception of the patient or the healthcare staff, and result in a decrease of expectations and engagement (Süssenbach et al., 2014), or generate a false sense of security that can lead to risks.

In our previous work (Lara et al., 2017; Casas et al., 2018c), we described the technical components of a sensor interface for obtaining patients' cardiovascular parameters (e.g., heart rate, recovery heart rate, and blood pressure) and spatiotemporal gait parameters (e.g., cadence, step length, and gait speed), in addition to the exercise intensity parameters (e.g., exertion level and treadmill inclination) and gaze direction indicating the cervical posture. We aimed to investigate the effects of using a socially assistive robot that uses these sensory values to give verbal feedback to the patient, in comparison to a Control condition, where the sensory values are only displayed on a tablet-based Graphical User Interface (GUI), i.e., no verbal feedback is given to the patient. Initial user studies (Lara et al., 2017; Casas et al., 2018a,b; Casas et al., 2018c; Casas et al., 2020; Aguirre et al., 2020; Irfan et al., 2020) validated the sensor interface and the robot within the laboratory and clinical settings based on case studies. In Casas et al. (2019), we compared the perceptions of the patients that have conducted the cardiac rehabilitation programme with the robot to the expectations of the patients that have not interacted with the robot through a control group. The results showed a significant increase in the perceptions of the robot's usefulness, sociability, and safety for the robot condition, in comparison to the expectations of the control group. In addition, patients in the robot condition expressed the desire to interact with the robot again. They also commented on its benefits on their progress during the sessions. Besides, a focus group was formed to evaluate the perceptions of the clinicians on the CR programme supported by the robot. After the initial questionnaire and discussion, the robot's capabilities were presented. The demonstration improved the perceptions of the clinicians for the robot's usefulness and safety, and facilitated trust to the system. The clinicians found the continuous monitoring aspect of the robot to be helpful in the case of emergencies and for applying high-intensity parameters during the training, in addition to helping them focus more on the needs of the other patients. Consequently, in this work, we present the perception of the clinicians who have worked with the robot and the findings based on the patients' physiological progress and interactions with the robot.

\section{PATIENT-ROBOT INTERFACE DESCRIPTION}

To evaluate the effect of a socially assistive robot in cardiac rehabilitation, this study compares two conditions: Control and Robot. We used a NAO robot from Softbank Europe ${ }^{2}$, a social robot widely used in human-robot interaction research. A patient-robot interface based on two modules was developed, as previously described in Lara et al. (2017). The first module includes the sensor interface that allows physiological parameters monitoring, and the second module, the robot module is in charge of the interaction and the social component, i.e., motivation and immediate feedback throughout the exercise.

\subsection{Sensor Interface}

As shown in Figure 1, a set of sensors were integrated to measure each parameter. The details of each parameter are explained in this section.

\subsubsection{Cardiovascular Parameters}

In cardiac physiology, several physical parameters are useful for studying the activity and regulation of the heart (Aamot et al., 2014). In CR, there is an increased interest to measure these parameters as they reflect the performance and progression of the patient. Consequently, our study analyses these parameters at different phases of the exercise:

\footnotetext{
${ }^{2}$ https://www.softbankrobotics.com/emea/en/nao.
} 


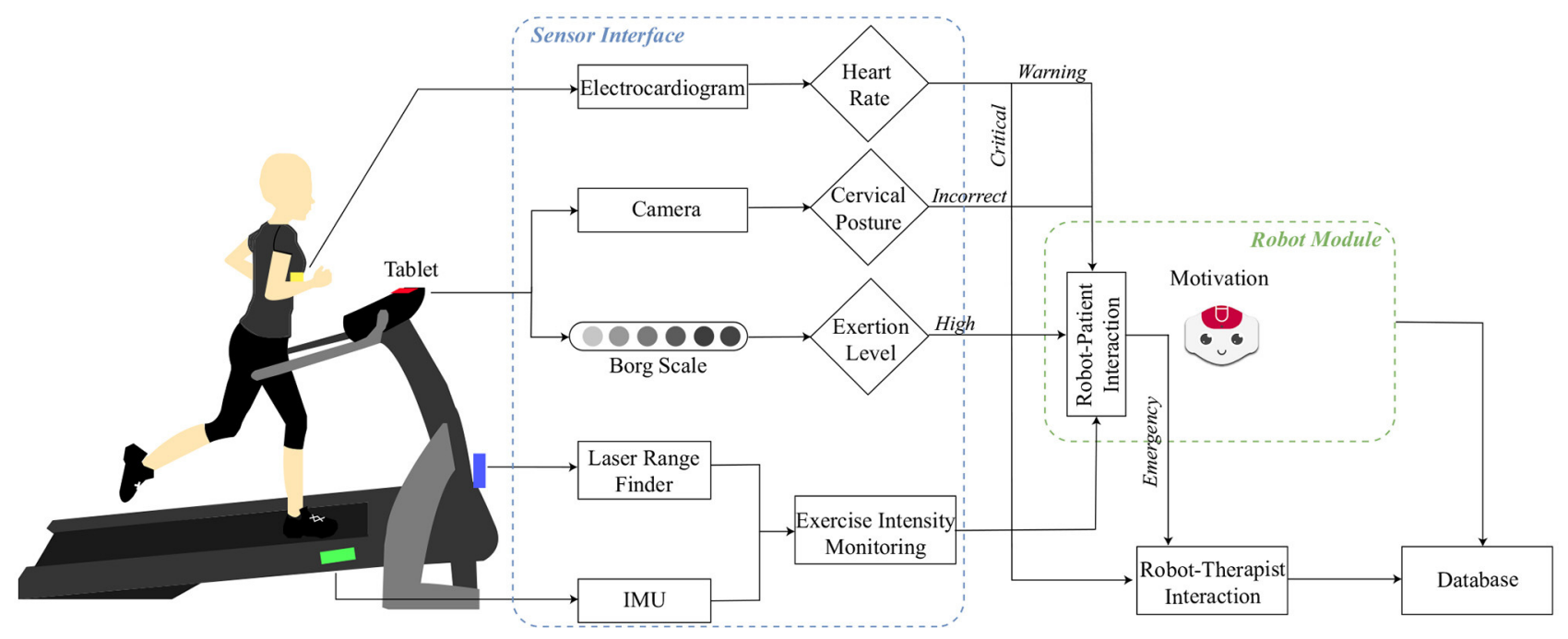

FIGURE 1 | A diagram of the patient-robot interface used in the cardiac rehabilitation programme.

- Training Heart Rate (THR) [bpm]: The average heart rate obtained during the treadmill exercise (15-20 min). The heart rate values were measured using an electrocardiogram Zephyr HxM sensor ${ }^{3}$ placed on the patient's chest.

- Recovery Heart Rate (RHR) [bpm/bpm]: This value represents the difference between the patient's $T H R$ and the average heart rate 1 -min post-training (average of 60 values $^{4}$ ) (Equation 1):

$$
R H R=T H R-H R_{\text {post-training }}
$$

To reduce the subjectivity of the measurements that change between the patients and increase the homogeneity, the RHR is normalized with the initial resting heart rate $(I H R)$, which was taken by the clinicians when the patient arrives in the clinic (Equation 2).

$$
R_{H} R_{\text {normalized }}=R H R / I H R
$$

\subsubsection{Gait Spatiotemporal Parameters}

Healthy gait is described as a series of rhythmical, alternating movements of the trunk and limbs, which results in the progression of the center of gravity and the body (AposTherapy, 2021). The patient's gait performance is analyzed by the gait components, which can be categorized under the following distance measurements (spatial) and time (temporal parameters):

- Cadence [steps/min]: The total number of full cycles taken within a given period (Thompson, 2002).

- Step Length [m]: The distance between the point of initial contact of one foot and the initial contact of the opposite foot (Thompson, 2002).

\footnotetext{
${ }^{3}$ Medtronic, New Zealand, https://www.zephyranywhere.com/.

${ }^{4}$ The sample frequency of acquisition corresponds to $1 \mathrm{~Hz}, 60$ values represent 1 min data.
}

- Gait Speed [mph]: This variable refers to the normal walking speed adopted by a person in everyday life (Thompson, 2002). Also, this variable represents the treadmill's speed.

In this case, a Hokuyo-URG 04LX-UG015 Laser Range Finder (LRF) was used to acquire these parameters during the session.

\subsubsection{Cervical Posture}

Cervical posture corresponds to the flexion of lower cervical vertebrae and their inclination (Shafer, 1987), which corresponds to the head inclination in sagittal, coronal and transverse planes. To measure this parameter the front camera of the tablet ${ }^{6}$ placed on the treadmill screen; and a head gaze estimator (Lemaignan et al., 2016) were used. During the exercise, a correct cervical posture is achieved when the patient looks straight ahead (i.e., the head gaze vector's vertical component is above 5.73 degrees). As the CR sessions are performed on a treadmill, the proper posture is essential to avoid dizziness, falls and nausea (Martin and McConahay, 1972).

\subsubsection{Physical Activity Intensity Parameters}

Three indicators were used to measure the intensity of the exercise: (1) The inclination of the treadmill, (2) the perceived exertion of the patient, and (3) the treadmill's speed ${ }^{7}$. An Inertial Measurement Unit MPU9150 ${ }^{8}$ was placed on the treadmill to measure the inclination, in a range of 0 and 5 degrees angle. The perceived exertion was measured using the Borg Scale (Borg, 1998). The Borg Scale is a subjective measurement commonly used in cardiac rehabilitation (Aamot et al., 2014) that allows the evaluation of the effort and intensity made by a patient during the exercise. At Fundación Cardioinfantil-Instituto de Cardiología

\footnotetext{
${ }^{5}$ Hokuyo, Japan, https://www.hokuyo-aut.jp/.

${ }^{6}$ Microsoft, USA, https://microsoft.com/es-es/surface.

${ }^{7}$ Corresponding to the patient's gait speed explained above.

${ }^{8}$ InvenSense, USA, https://invensense.tdk.com/.
} 


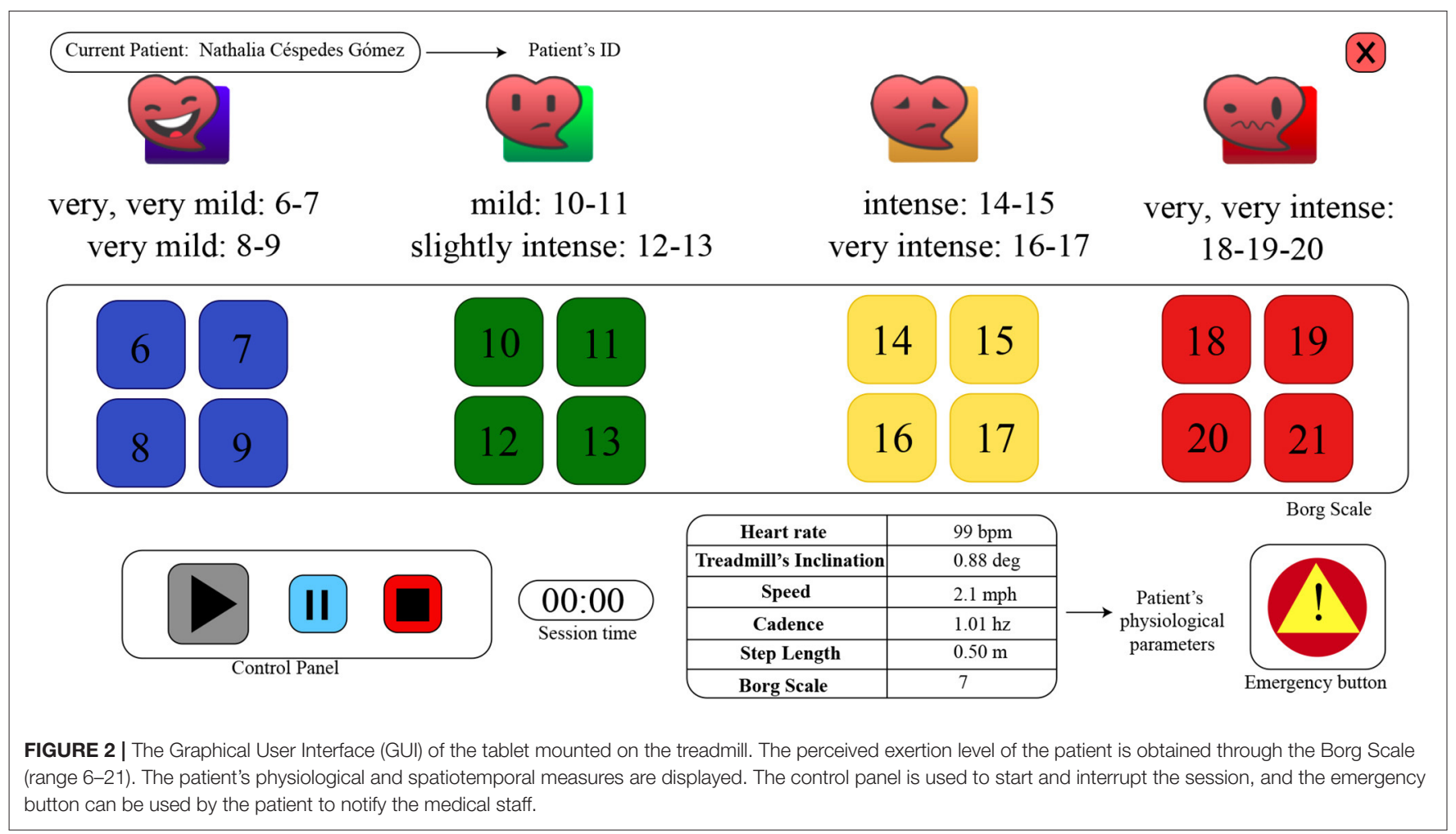

the scale is between 6 and 20, where 6 corresponds to a very low level of exertion and 20 corresponds to a very high level of exertion. The safe range is considered to be $6-12$ by the medical staff (Figure 2).

\subsubsection{Borg Scale Response Time}

Borg Scale is periodically (every $7 \mathrm{~min}$ ) requested through the tablet interface, as shown in Figure 2. For the Control condition, an audible signal is given, along with a change of color in the tablet interface (i.e., the Borg scale is grayed out, when inactive), whereas, the robot verbally asked the patients to enter their exertion level on the tablet each time. The Borg Scale Response time [s], represents the time taken by the patient to answer a Borg Scale request made by the robot. In the case of the control group, this variable was not measured as the interface did not register the events when the request was activated and responded.

The patient-robot interface was validated in an initial study under laboratory conditions (Lara et al., 2017). Subsequently, the system was deployed for clinical set-up lasting 18 weeks (36 sessions) at the Fundación Cardioinfantil-Instituto de Cardiología clinic (Figure 3) for 2.5 years.

\subsection{Robot Module}

The Robot Module focuses on interaction with the user. This interaction is divided into three states: (1) Motivational support, (2) Performance monitoring, and (3) Online feedback. A session with the robot starts with an initial greeting, where the robot makes an announcement of the intensity that will be performed during the session (i.e., treadmill inclination and speed). Performance monitoring starts when the patient starts

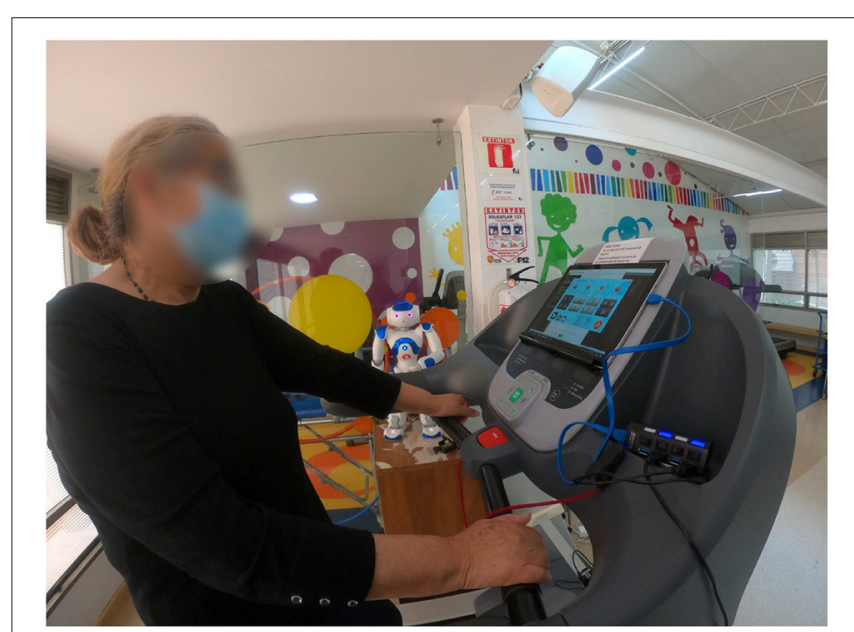

FIGURE 3 | Setup of the experiment with the NAO robot (SoftBank Robotics Europe) and the tablet interface for cardiac rehabilitation at Fundación Cardioinfantil-Instituto de Cardiología clinic.

their exercise on the treadmill. Motivational support occurs periodically every $5 \mathrm{~min}$ during the session. The robot encourages the patients through verbal phrases (e.g., "You can do it!", "You are doing great"” among others), accompanied by non-verbal gestures that are synchronized to verbal speech through the animated speech module ${ }^{9}$ of the NAO robot, in addition to

\footnotetext{
${ }^{9}$ http://doc.aldebaran.com/2-5/naoqi/audio/alanimatedspeech.html
} 


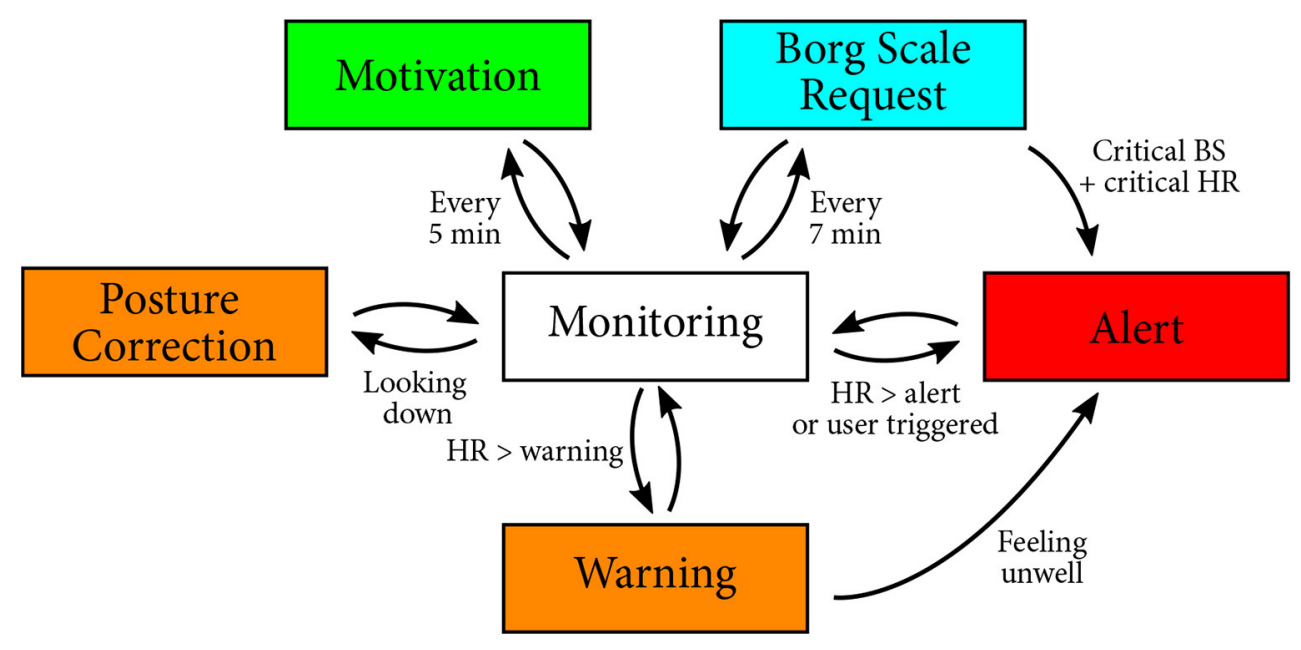

FIGURE 4 | Finite state machine presenting the different transitions possible during the monitoring phase.

face tracking for gaze maintenance. Similarly, the Borg scale was requested every $7 \mathrm{~min}$ to ensure that the exertion level remains within the acceptable range. During this monitoring state, sensory information is analyzed. Depending on the values given by each sensor, the current state can activate the online feedback state or remain in the same state. When the online feedback is activated, the robot reacts differently based on the event (Casas et al., 2018c): (1) high or critical heart rate, (2) high exertion level, and (3) incorrect cervical posture (See Figure 4). Additionally, we added a cooldown period of 3 min after feedback was provided to prevent the robot from repeating the same feedback too often. When an alert is triggered (i.e., for critical heart rate or by the patient through the tablet interface), the robot calls for help to the medical staff verbally ("Your heart rate is too high, I am calling for help. Doctor, could you please come here?") with a waving gesture. The alert will be repeated until the medical staff touches the head of the robot to notify their intervention.

\subsubsection{Heart Rate Feedback}

This feedback is given by the robot when the heart rate exceeds the warning or critical thresholds. These thresholds are determined before the start of the session by a physiatrist and entered on the tablet GUI. The warning threshold corresponds to the maximum of the determined healthy range (i.e., High HR Warning). While reaching this value is not critical, it may suggest that the patient is experiencing difficulties with the session intensity. Hence, the robot asks verbally for feedback from the patient through the GUI to report whether they are feeling well. If the patient is not feeling well, the robot alerts the medical staff verbally and non-verbally (i.e., arm gesture). The critical threshold corresponds to the maximum heart rate allowed for the patient (i.e., Call Medical Staff Alert), calculated by the medical staff using the Karvonen formula (She et al., 2014) (Equation 3), where $H R_{\text {optimal }}$ represents the optimal heart rate during the exercise, $H R_{\max }$ is the maximum $\mathrm{HR}$ allowed for the patient, $I H R$ means the resting $\mathrm{HR}$, and \%Effort represents the percentage of desired exercise intensity. Exceeding the $H R_{\text {optimal }}$ level may result in a complication; hence, it is vital to promptly alert the medical staff when this value is reached. Correspondingly, the robot directly alerts the medical staff without confirmation from the patient.

$$
H R_{\text {optimal }}=\left[\left(H R_{\max }-I H R\right) * \% \text { Effort }\right]+I H R
$$

\subsubsection{Exertion Level Feedback}

This feedback is given when the patient enters the Borg Scale in the tablet. According to the value of the perceived exertion level, three types of robot behaviors are activated during the session: (1) If the Borg scale is on a normal range, the robot thanks the patient, (2) if the patient enters a critical Borg scale (above 12), but the current heart rate is in a healthy range, the robot asks for a confirmation of the Borg scale, and (3) when both the Borg scale and the heart rate are critical, the robot alerts the medical staff.

\subsubsection{Cervical Posture Feedback}

This feedback is given by the robot when the patient is not looking straight (e.g., looking down at the treadmill or looking sideways). The patient needs to maintain a good cervical posture, i.e., the patient should look straight ahead, to avoid dizziness and other risk factors (e.g., falls). In this case, the robot gives verbal feedback to the patient, asking to maintain a straight posture.

\section{METHODOLOGY}

This section describes the protocol carried out to evaluate the effects of a social robot in cardiac rehabilitation. A longitudinal study was conducted lasting 2.5 years for the outpatient phase (II) of cardiac rehabilitation, corresponding to 36 sessions that generally corresponds to 18 weeks if the patient comes twice a week, as prescribed. 


\subsection{Research Question}

Our study aims to answer the research question, What are the benefits of using a socially assistive robot for long-term cardiac rehabilitation? Based on the previous literature outlined in section 2, we expect an improvement in the motivation, which could improve the adherence to the CR programme and the task performance (i.e., cardiovascular functioning and recovery). Moreover, the loss of interest that may arise in long-term interactions after the novelty effect wears off, can cause a decay in the interaction with the robot and the patient perceptions. Additionally, the collaboration of the medical staff is fundamental in ensuring a rapid intervention in case of emergencies. Thus, their perception of the robot and feedback are central to applications of the robot in clinical scenarios. Correspondingly, in collaboration with the medical staff, we designed a clinical study that incorporates and assesses these elements.

\subsection{Conditions}

To observe the effects of the social robot, two conditions were designed for the study. The details of each condition are described below:

- Control condition: Within this condition, the participants perform a conventional session of cardiac rehabilitation. As this condition is considered as a baseline, the patients of the Control group are monitored only by the sensor interface. In other words, the patient interacts with the tablet only to enter the Borg Scale without the presence of the robot. Therefore, the patients of the Control group do not receive any type of feedback or motivation provided by the robot. However, the physiological parameters (i.e., patient's heart rate, gait speed, cadence and step length, treadmill inclination and the previous self-reported Borg scale value) of the patients are displayed on the tablet GUI to inform the medical staff, as shown in Figure 2.

- Robot condition: This condition integrates the complete described system, that is the patient-robot interface. As shown in Figure 3, the fully-autonomous robot is placed next to the patient below the eye level, to enable the visual attention on the robot's behaviors and feedback. Once the session begins, the robot provides monitoring, feedback and motivation to the patient during the exercise.

The sensor interface and the robot operated fully autonomously. Nonetheless, an experimenter was present in the room during the sessions in both conditions for safety purposes, and only interfered with the session in the case of system failures.

\subsection{Experimental Criteria}

- Inclusion criteria: The patients considered within this study were those who started phase II (36 sessions) of the cardiac rehabilitation programme that would attend twice a week to the sessions. The patients that are over 25 years old ${ }^{10}$ with acute myocardial infarction (AMI), percutaneous coronary

\footnotetext{
${ }^{10}$ The patient's age was determined by the medical staff due to the common minimum age of the patients who begin the CR programme.
}

intervention, coronary artery bypass graft (CABG), valve replacement, ischemic heart disease and hypertension and ejection fraction greater than $40 \%$ were recruited. Also, these patients have to be able to perform treadmill exercise.

- Exclusion criteria: Considering the requirements of the system, our system may pose limitations on the patients in the case of any visual, auditive or cognitive impairment that impede the manipulation and correct understanding of the system. Thus, we could not include those patients in the study. The patients that present a different cardiovascular pathology mentioned in the inclusion criteria were also not considered for the experiment.

- Dropout and Incomplete criteria: Initially, 18 weeks were considered as the CR programme duration, in which patients would attend twice per week. However, some patients missed CR sessions; consequently, this initial policy resulted in a shorter exercise session for the patients (23-33 sessions). Hence, the policy was reviewed in 2018 to improve the CR programme offered to the patients for lasting 36 sessions instead. Thus, a "drop-out" is considered when the patient does not attend three sessions in a row, without justification. In this case, the patient is dropped from the study; however, the patient could continue the CR programme without the robot assistance. Furthermore, an incomplete CR programme is considered when patients that could not complete the CR programme due to a critical health condition, funding or COVID-19 outbreak, since these reasons are beyond their control.

\subsection{Participants and Demographic Data}

According to the experimental design of the study, we recruited 30 patients (15 per condition). However, as stated in the introduction, the adherence to cardiac rehabilitation is low due to several factors. Consequently, we had 9 patients in the Control condition (ages between 44 and 70) and 11 patients in the Robot condition (ages between 43 and 80), who actively participated in the rehabilitation and completed the outpatient phase (II) of the cardiac rehabilitation programme. Table 1 shows the demographic data of these patients. The first four sessions of 4 patients ( 2 in Control, 2 in Robot) and the complete CR programme of 6 patients ( 3 in Control, 3 in Robot) were presented in prior work as case studies (Casas et al., 2018a, 2020). The data for these patients were included in the analysis for this work.

The patients' schedules were arranged such that during a CR session with 20 patients, only one subject from the study participated in the session. In other words, 19 other patients present at the CR session were not part of the study, and received conventional CR programme, where the medical staff obtained their measures. While around 200 patients receive CR per day, due to these scheduling restrictions, the availability of the experimenters, and the participation in the study, only 3-5 to patients from the study attended per day.

\subsection{Measures}

To answer our research question, we analyzed the patients' adherence, physiological parameters, how they interacted with the robot and their perceptions of the robot as well as the 
perceptions of the clinicians that were part of the CR programme with the socially assistive robot.

\subsubsection{Dropout Rate}

The dropout rate corresponds to the ratio of patients that dropped out based on the withdrawal criteria, to the total number of patients that were recruited in the study.

\subsubsection{Physiological Parameters}

The main physiological parameters evaluated during the study are the Cardiovascular and Physical activity intensity parameters (i.e., RHR, THR, and Borg scale). A higher RHR would suggest an improvement in the health status of the patient, whereas the $T H R$ and Borg scale would show the difficulties experienced in the sessions.

To analyse the data, six stages were proposed. Each stage contains 6 sessions, in total corresponding to the complete CR programme. The analysis per stage was performed to reduce the data and reduce the intrasubject variability.

\subsubsection{Interaction With the Robot}

Because feedback was not provided in the Control condition, the gaze direction of the patient was only estimated in the Robot condition. In addition, Borg Scale Response Time was only measured for the Robot group. Moreover, the counts for the Heart Rate Feedback (i.e., High HR Warning and Call Medical Staff Alert) were also measured.

\subsubsection{Perception of the Robot}

As discussed in section 2, using a socially assistive robot for the CR sessions affects not only the patient but also the medical staff. Especially in our study, the collaboration of the medical staff was essential. Hence, the medical staff that interacted with the robot throughout the experiment, which lasted 2.5 years, was interviewed (one physiatrist, one nurse and one physical therapist), to collect their perspectives of the robot and the study. A semi-structured interview was performed online and lasted less than $30 \mathrm{~min}$. Questions regarding their experience (e.g., "Did you know anything about social robotics before you started the study?", "Were the expectations you had of the robot fulfilled?"), attitudes (e.g., "Did you find useful the robot in the programme?", "Which aspects (negative/positive) regarding the robot assistance could you highlight?", "Did the robot change in someway the conventional CR session?"), and opportunities (e.g., "Would you continue using the robot?", "If you have the opportunity, which features would you add to the social robot?", "Would you recommend the robot to other healthcare colleagues?").

Additionally, an adapted Unified Theory of Acceptance and the Use of Technology (UTAUT) questionnaire (Venkatesh et al., 2003; Casas et al., 2019) was applied to the patients to evaluate their perceptions (or expectations) at the end of their CR programme. The results were previously analyzed in detail in Casas et al. (2019), which showed that the patients perceived the robot positively in terms of ease of use, utility and safety after the completing the CR programme, as briefly outlined in section 2 .

\subsection{Statistical Analysis}

The data were analyzed within six stages to reduce the intrasubject variability caused by external factors (e.g., illness, tiredness level prior to the session), as suggested by the medical staff. Each stage consists of 6 sessions, in total corresponding to the complete CR programme.

The data for physiological progress, cervical posture and the interactions with the robot are not normally distributed $(p<0.00001$ in Shapiro-Wilk normality test on residuals and residuals divert from linear reference lines in visual inspection), and the homogeneity of variances assumptions are violated $(p<$ 0.05 in Levene's test and/or Box's $M$-test) for training heart rate, inclination and the medical alert. Due to the dropouts and the incomplete CR as previously described, we have unbalanced (non-equal group sizes) data. Moreover, some of the patients completed the CR programme earlier (due to the change in the experimental criteria) and we experienced sensor failures within some of the sessions, hence, we have incomplete (missing) data. Thus, we apply Johansen's (Johansen, 1980) general formulation of Welch (Welch, 1938)-James (James, 1951)'s statistic with Approximate Degrees of Freedom (ADF) (Welch, 1951; Keselman et al., 2003; Villacorta, 2017), which is suitable for non-parametric repeated measures and two-way mixed design (within-subject factor is stages and between-subject factor is condition). The results of the two-way mixed design are reported for stage and condition effects, and their interaction (whether the effect of condition, depends on the stage). We also report the significant differences between conditions for each stage, and the pairwise significant differences between stages for each condition for analyzing longitudinal effects. Hochberg correction for multiple comparisons (pairwise tests) and Least-Squares Estimators (i.e., trimming is not applied on the data) are used, which are the default parameters of the welchADF test in $\mathrm{R}$ (Villacorta, 2017) ${ }^{11}$. The results are reported in the format $T_{W J}\left(d f_{1}, d f_{2}\right)$ for the Welch-James ADF test statistic, where $d f_{1}$ and $d f_{2}$ are the approximate degrees of freedom for the numerator and denominator, in addition to the $p$-values. The effect sizes are reported based on Glass's $\delta$ (Glass et al., 1981; Keselman et al., 2003; Villacorta, 2017) for pairwise comparisons. Note that in contrast to Cohen (1988) and Glass et al. (1981) are critical for classifying effect sizes as "small," "medium," and "large," as the practical importance of an effect depends on the context (i.e., relative costs and benefits) and even small effect sizes can make a substantial difference. Thus, the effect sizes should instead be used to evaluate the consistency and the magnitude of a particular phenomenon across different studies in the literature. Negative effect size denotes that the mean in group 2 (e.g., Robot condition or stage 2) is lower than mean in group 1 (e.g., Control condition or stage 1), whereas, positive effect size denotes that the mean is higher. Moreover, Chi-Square $Z$-test is applied on recovery heart rate $(R H R)$ to determine whether the observed frequencies markedly differ from the frequencies that we would expect by chance.

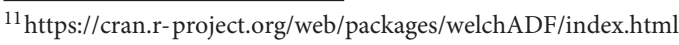


TABLE 1 | Demographic data of the patients who have finished the outpatient phase (II) of the CR programme within the study.

\begin{tabular}{lcc}
\hline & Control & Robot \\
\hline Participants & 9 & 11 \\
Gender & 9 males & 10 males, 1 female \\
Age (years), mean (SD) & $56.6(7.8)$ & $55.7(11.2)$ \\
Body Mass Index, mean (SD) & $26.2(2.6)$ & $29.2(3.9)$ \\
- Obese & $0.0 \%$ & $54.5 \%$ \\
- Overweight & $66.7 \%$ & $36.4 \%$ \\
- Healthy weight & $33.3 \%$ & $9.1 \%$ \\
Level of education & & \\
- Elementary school degree & $22.2 \%$ & $18.2 \%$ \\
- High school degree & $22.2 \%$ & $27.3 \%$ \\
- Technologist & $0.0 \%$ & $18.2 \%$ \\
- Bachelor's studies/degree & $55.6 \%$ & $18.2 \%$ \\
- Postgraduate studies/degree & $0.0 \%$ & $18.2 \%$ \\
\hline
\end{tabular}

\section{RESULTS}

Our study aims to improve adherence rate with the use of a socially assistive robot. Correspondingly, we report the patient attendance in section 5.1. In section 5.2, we evaluate the effects of SAR on the physiological progress of the patients during the CR programme of 36 sessions, which was inspected in six stages (6 sessions each). Subsequently, we evaluate how the patients interacted with the robot (section 5.3), and the perception of the patients and the medical staff (section 5.4).

\subsection{Adherence}

Thirty patients were included in the 2.5 years of the clinical study, corresponding to 670 sessions. However, only 20 patients (Table 1) actively participated in the rehabilitation and completed the outpatient phase as established by the criteria in section 4.3. Figure 5 shows the adherence between the conditions. According to the results, six patients who were in the Control condition and four patients of the Robot condition did not complete phase II of CR. As it can be observed, these patients attended 11 sessions on average before withdrawing from the CR programme. The dropout rate between the conditions (33.3\% for Control, 20\% for Robot condition) shows that the adherence was lower in the Control condition than in the Robot condition. This outcome was highlighted during the clinicians' interview, where the physiatrist in charge of the rehabilitation expressed that the patients during conventional CR programme generally take more time to finish the CR. To obtain an unbiased (i.e., not affected by participation in a study) baseline adherence rate, we analyzed the medical records in the Fundación CardioinfantilInstituto de Cardiología clinic for $14 \mathrm{CR}$ patients that were not part of the study, which showed that patients take 5.7 months on average to finish the outpatient phase (II). In the Control condition, this rate was about 4.7 months on average, indicating that patients were committed to completing the programme due their participation in the study. Within the
Robot condition, this rate was lower, lasting 4.6 months on average. This initial result shows the potential of SAR to improve adherence, which is vital for patients to achieve a complete recovery (Ruano-Ravina et al., 2016).

\subsection{Physiological Progress}

The recovery heart rate $(R H R)$ is considered by the clinicians as the primary physiological parameter, as it determines the $\mathrm{CR}$ progress. From a clinical point of view, the recovery of the patient changes as the physical exercise influences the patient's cardiovascular functioning. Hence, it is expected that this value increases along with the rehabilitation programme as this tendency elucidate a healthy recovery. Figure 6 shows the comparison between RHR in both conditions. When performing the statistical test over the normalized $R H R$, the results highlight significant differences between stages $\left[T_{W J}(5,238)=\right.$ $11.02, p<0.001]$ showing the expected behavior of the RHR throughout the CR programme (Figure 6). Although, the normalized RHRs present significant differences between conditions $\left[T_{W J}(1,362)=12.35, p<0.001\right]$, the analysis per stage only show differences for the stage $3(p=0.02, \delta=0.47)$ and stage $6(p=0.01, \delta=0.64)$. No significant differences were found for the "interaction" between conditions and stages $\left[T_{W J}(5,238)=1.39, p=0.23\right]$. Moreover, Table 2 shows the results regarding the differences between stages within the same condition. As it can be observed, the Robot condition exhibits significant differences compared to the initial stage after stage 3 with greater increments than the Control condition. This result may arise from the decrease of recovery heart rate $(R H R)$ of two patients in the Control condition. No other significant differences were observed between consecutive stages, which suggests that the CR programme has rapid positive effects on patient recovery.

According to the literature, an $R H R$ greater than $22 \mathrm{bpm}$ represents a healthy value and a successful rehabilitation process (Carnethon et al., 2015). Thus, a Chi-Square $Z$-test was applied to observe the clinical relevance of the RHR between the groups. The results show that there exists a significant difference between the Control and Robot groups $\left[z_{(1, N=114)}=\right.$ $-1.82, p=0.03$ ]. As can be seen in Table 3, a higher number of patients exceeded the threshold in the Robot group, showing that the patients in the Robot condition have a better physical activity performance.

As CR progresses it is expected that the patients' physiological performance changes, as well as the physical exercise intensity. For instance, the Borg Scale (Figure 7A) presents differences between stages independently of the condition $\left[T_{W J}(5,277)=\right.$ $3.32, p=0.006]$, demonstrating that patients perceive exertion differently from one stage to another as the exercise intensity is increasing throughout the programme, especially between the initial stage and the later stages (stage $4, p=0.04, \delta=0.79$, and stage $5, p=0.004, \delta=1.0$ ). The comparison between the Control and the Robot conditions did not present significant differences overall $\left[T_{W J}(1,512)=0.28, p=0.59\right]$. However, there exists significant differences between the conditions for stage $5(p=0.003, \delta=0.59)$. From the longitudinal perspective (comparison between stages of the same condition), the Control 

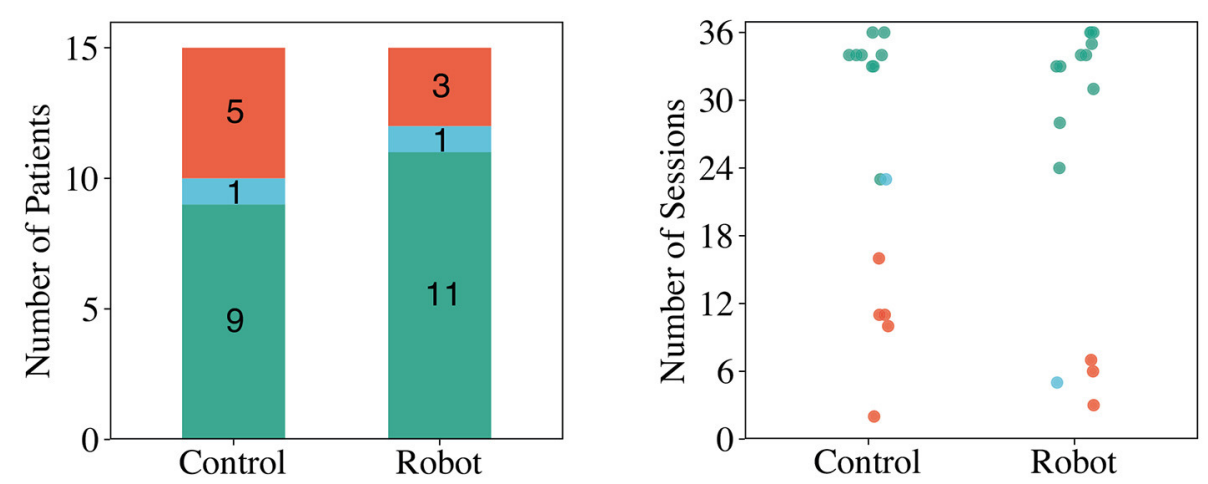

Therapy Status $\square$ Dropout $\square$ Incomplete $\square$ Complete

FIGURE 5 | The CR programme status of the users in the Control and Robot conditions: complete refers to the completed cardiac rehabilitation programme as determined by the clinicians; incomplete is when patients need to stop the session due to reasons beyond their control, and dropout refers to dropping out of the study or not attending three sessions in a row without a justification.

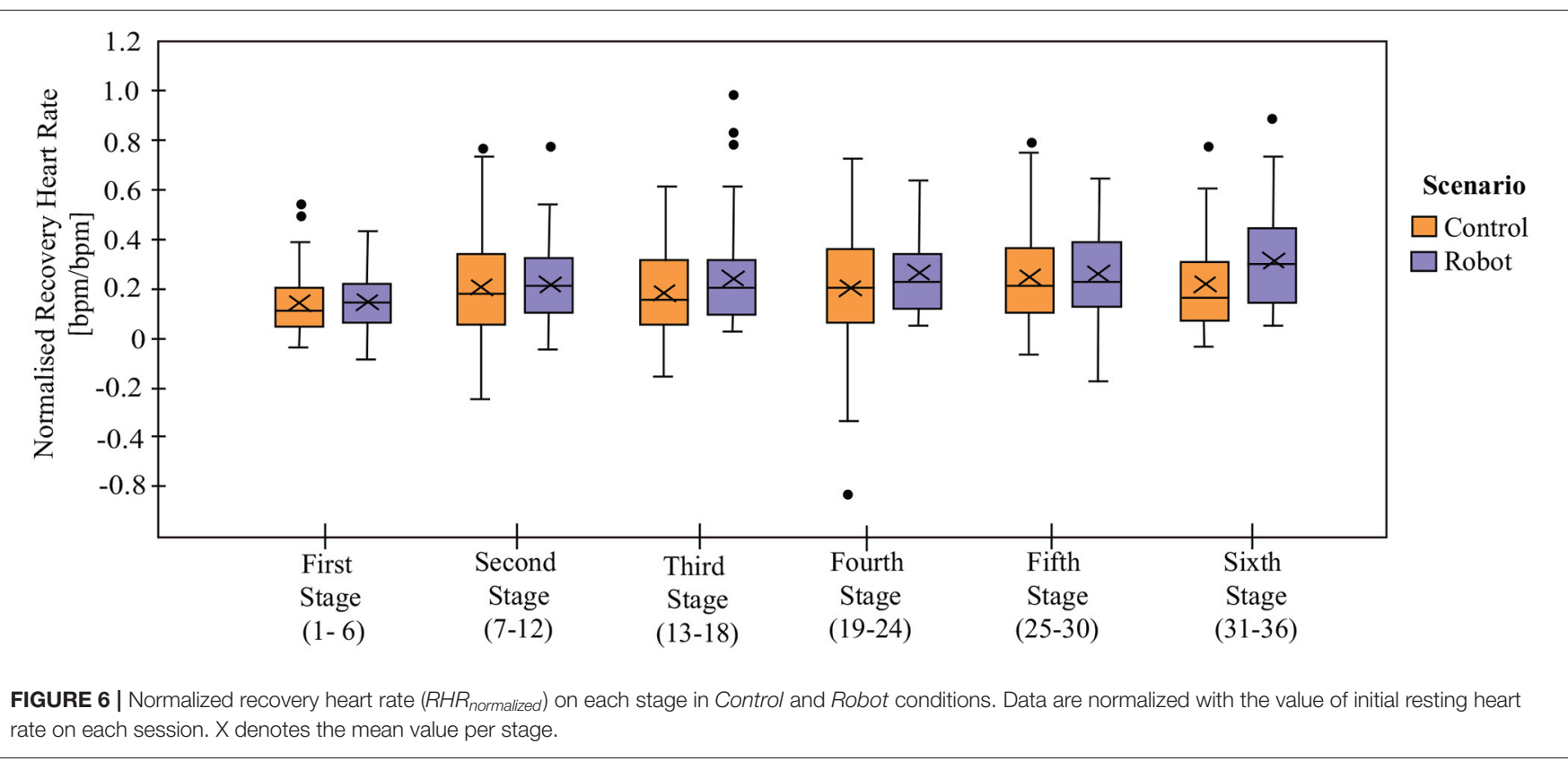

TABLE 2 | Welch-James ADF results ( $p$-value and effect size in parentheses) for normalized Recovery Heart Rate $\left(R H R_{\text {normalized }}\right)$.

\begin{tabular}{|c|c|c|c|c|}
\hline & Control & Increment \% & Robot & Increment \% \\
\hline Stage 1/Stage 2 & $p=0.27(0.47)$ & 49.35 & $p=0.052(0.54)$ & 54.89 \\
\hline Stage 1/Stage 3 & $p=0.72(0.37)$ & 34.24 & $p=0.002(0.75)$ & 82.43 \\
\hline Stage 1/Stage 4 & $p=0.11(0.55)$ & 48.55 & $p<0.001(0.99)$ & 98.96 \\
\hline Stage 1/Stage 5 & $p=0.01(0.75)$ & 92.44 & $p<0.001(0.90)$ & 96.93 \\
\hline Stage 1/Stage 6 & $p=0.85(0.40)$ & 68.24 & $p<0.001(1.14)$ & 135.82 \\
\hline
\end{tabular}

Significant differences $(p<0.05)$ are highlighted in bold. Consecutive stages and other pairwise comparisons do not exhibit any significant differences.

group presents significant difference between stages 1 and $4(p=$ $0.004, \delta=0.73)$, stages 1 and $5(p<0.001, \delta=1.12)$, whereas, none of the stages present significant differences in the Robot condition. In agreement with these results, the "interaction" of stage and condition for the Borg Scale present significant differences $\left[T_{W J}(5,277)=2.89, p=0.01\right]$. 
TABLE 3 | Chi-square Z-test for the Recovery Heart Rate (RHR) between Control and Robot conditions.

\begin{tabular}{lcc}
\hline & \multicolumn{2}{c}{ Frequency per condition } \\
\cline { 2 - 3 } & Control & Robot \\
\hline$R H R>22$ & 9 & 20 \\
$R H R<22$ & 43 & 42 \\
Total & 52 & 62 \\
\hline
\end{tabular}

$z(1, N=114)=-1.82, p=0.033$

The physical activity parameters that determine the session exercise intensity are the Gait Speed (Figure 7B) and the Treadmill Inclination (Figure 7C). In both cases, the results show that there are significant differences between stages [Speed: $T_{W J}(5,256)=14.95, p<0.001$ and Inclination: $T_{W J}(5,233)=$ $4.92, p<0.001]$, demonstrating that the physical exercise parameters change (increase or decrease) across the duration of the CR programme based on the patient's performance and the physiological progress during the sessions. The difference between conditions did not show significant differences [Speed: $T_{W J}(1,485)=0.59, p=0.44$ and Inclination $T_{W J}(1,381)=$ $3.46, p=0.06$ ] neither the comparison per stages [Speed: $p>0.05$ and Inclination: $p>0.05$ ], which means that the physical activity parameters were prescribed homogeneously for both groups. For the comparisons between the stages within the same condition, the Speed presents significant differences in both groups as shown in Table 4. The Inclination presents no significant differences between stages in the Robot condition, whereas there are significant differences in the Control condition between stages 1 and $5(p=0.003, \delta=0.83)$ and stages 1 and 6 $(p=0.004, \delta=0.98)$.

In accordance with the previous results, there are significant differences between stages for the Training Heart Rate (THR) $\left[T_{W J}(5,194)=3.31, p=0.007\right]$, which is to be expected as the session exercise intensity (determined by the speed and inclination) is changing throughout the $\mathrm{CR}$ programme (Figure 7D). While significant differences are found between the conditions $\left[T_{W J}(1,309)=4.35, p=0.04\right]$, there does not exist any significant differences between the conditions per stage $(p>0.05)$. Moreover, there is a lack of significant differences on the interaction of stage and condition $\left[T_{W J}(5,194)=\right.$ $0.76, p=0.58]$. When the differences between stages are analyzed separately per condition, the THRs in the subsequent stages are found to be significantly different than the initial stage for the Robot condition, as presented in Table 5, whereas the stages were found to be significantly equivalent for the Control condition.

\subsection{Interaction With the Robot}

Regarding the interaction with the robot, four indicators were measured for the Robot condition: Cervical Posture Feedback (Figure 8), Borg Scale Response Time (Figure 9), High HR Warning and Call Medical Staff Alert (Figure 10). The results obtained after the statistical analysis elucidate that there are no significant differences between stages for most of the interaction indicators [Borg Scale Response Time: $T_{W J}(5,149)=1.17, p=$
0.33, Cervical Posture Feedback: $T_{W J}(5,148)=1.5, p=0.19$, and High HR warning: $\left.T_{W J}(5,154)=1.05, p=0.39\right]$. These results suggest that the patients tend to maintain their interaction behavior throughout the duration of the CR programme. The similar posture corrections between stages indicate that the patients made efforts to keep a healthy posture. Moreover, the results indicate that the patients continued to respond rapidly to the robot's requests throughout the long-term CR programme.

In contrast, the Call Medical Staff Alerts present significant differences between stages $\left[T_{W J}(5,132)=9.67, p<0.001\right]$. As visible in Figure 11, the significant differences per stages were founded between stages 1 and $3(p=0.02, \delta=0.77)$, stages 1 and $4(p=0.005, \delta=0.8)$, stages 1 and $6(p=0.02, \delta=1.02)$. Most of these results are due to sessions where this alert was triggered for a high number of times due to malfunctioning of the robot. While this limitation may have caused false positives, this alarm is very important and vital to the clinicians for immediately detecting an emergency (e.g., symptoms of dizziness or abnormal blood pressure), such that the patient could be supported in a rapid manner (Irfan et al., 2020).

\subsection{Perception of the Robot}

The feedback from the clinicians was positive overall. Most of the clinicians highlighted that the $\mathrm{CR}$ felt more secure using the robot due to the continuous monitoring: "Within conventional sessions our resources are limited, however, the help of the robot enhances the supervision of the session," "I was more aware of the patient's progress thanks to the robot feedback," and "I feel more secure due to the robot's continuous monitoring".

The feedback of the patients during the study included (Casas et al., 2019): "I feel more compelled to do the exercise because the robot is monitoring me," "I was very insecure at the beginning of the rehabilitation and thanks to the robot I got confidence," "I want to come to my rehabilitation, I have the advantage that the robot watches over my health status every second and I feel more secure." The clinicians agreed with the positive comments regarding the adherence. When they were asked about the robot's benefits, they commented as: "The patients who work along with the robot were more engaged with the CR programme," "The patients who work with the robot were more interested to know their own progress," and "The patients feel more secure with the robot monitoring, and they like the robot, so they have more trust in the rehabilitation."

Nonetheless, some of the patients commented on the lack of personalization of the robot: "I would like the robot to be more personalised," "I would like the interaction to be closer to the patient (e.g., be more sociable, perform reminders, use patients' name.)" and "The robot seems a little repetitive".

Finally, the patients and clinicians highlighted that they would like to continue using the robot in the future and they also recommended its use to other patients and healthcare partners during the rehabilitation procedure.

\section{DISCUSSION}

During 2.5 years of the study, 30 patients were recruited to perform CR programme within both conditions. However, only 20 patients completed the treatment. The results include the 


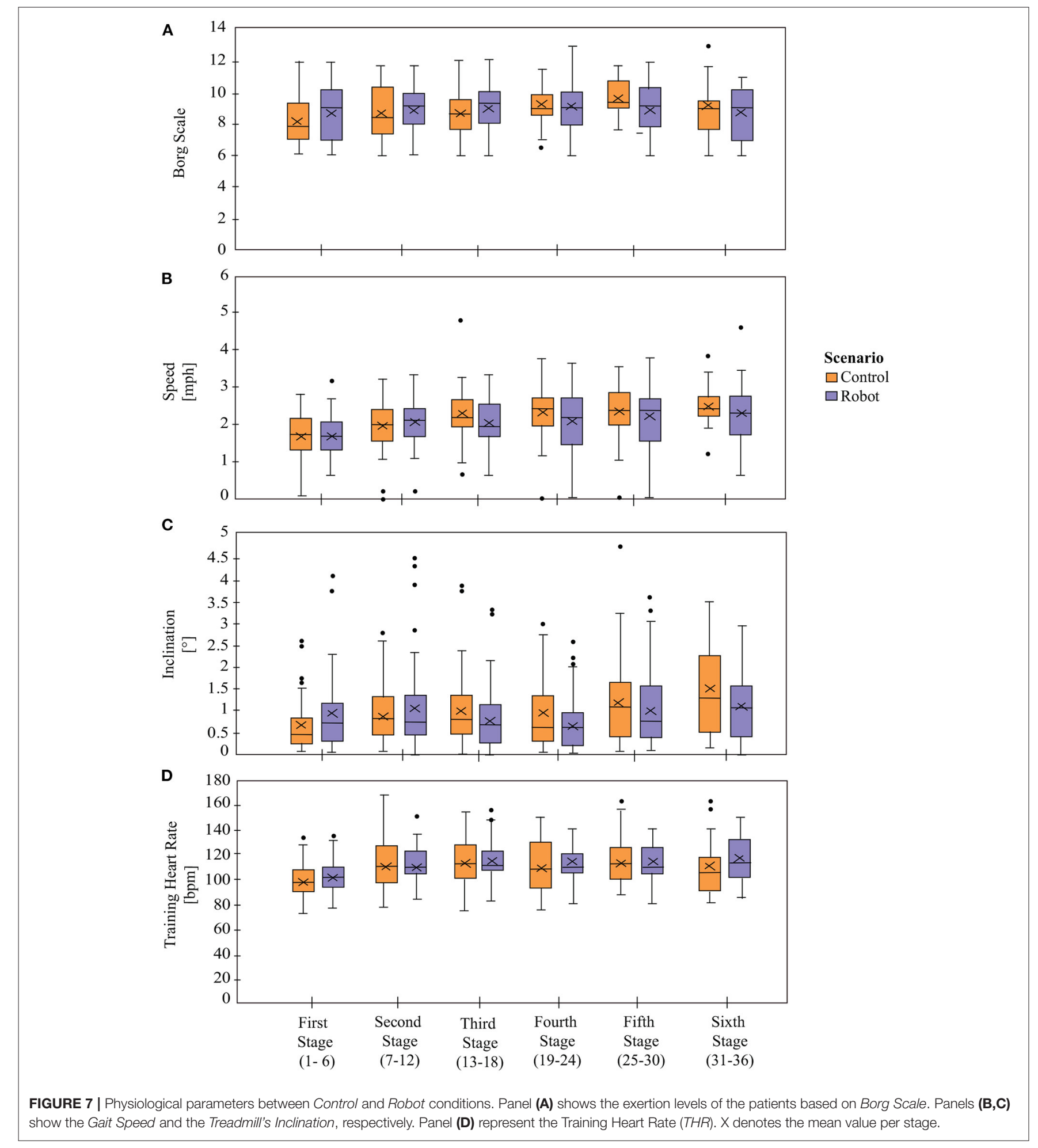

analysis of 20 patients ( 9 in the Control condition, and 11 in the Robot condition) who finished the treatment.

As mentioned in section 1 , several rehabilitation programmes present limitations on the adherence to the CR programme. This limitation is caused in part by the psycho-social factors, such as motivation, engagement and anxiety. In this study, the patients' assisted by the social robot had higher adherence to the CR programme, thus, we believe that the robot had a positive effect over the engagement of the patients during the rehabilitation programme due to the continuous monitoring, the 
TABLE 4 | Welch-James ADF results ( $p$-value and effect size in parentheses) for Gait Speed.

\begin{tabular}{|c|c|c|c|c|}
\hline & Control & Increment \% & Robot & Increment \% \\
\hline Stage 1/Stage 2 & $p=0.17(0.48)$ & 22.66 & $p=0.12(0.46)$ & 16.18 \\
\hline Stage 1/Stage 3 & $p<0.001(0.96)$ & 44.06 & $p=0.003(0.66)$ & 22.64 \\
\hline Stage 1/Stage 4 & $p=0.002(0.79)$ & 41.66 & $p=0.02(0.57)$ & 22.98 \\
\hline Stage 1/Stage 5 & $p<0.001(0.89)$ & 42.56 & $p=0.001(0.78)$ & 24.36 \\
\hline Stage 1/Stage 6 & $p<0.001(1.21)$ & 45.58 & $p=0.001(1.04)$ & 41.49 \\
\hline
\end{tabular}

Significant differences $(p<0.05)$ are highlighted in bold. Consecutive stages and other pairwise comparisons do not exhibit any significant differences.

TABLE 5 | Welch-James ADF results ( $p$-value and effect size in parentheses) for Training Heart Rate (THR).

\begin{tabular}{|c|c|c|c|c|}
\hline & Control & Increment \% & Robot & Increment \% \\
\hline Stage 1/Stage 2 & $p=0.41(0.44)$ & 9.48 & $p=0.03(0.55)$ & 8.72 \\
\hline Stage $1 /$ Stage 3 & $p=0.71(0.39)$ & 8.59 & $p=0.002(0.70)$ & 11.47 \\
\hline Stage $1 /$ Stage 5 & $p=0.21(0.52)$ & 12.53 & $p=0.01(0.63)$ & 10.71 \\
\hline Stage 1/Stage 6 & $p=0.99(0.16)$ & 6.18 & $p=0.01(0.77)$ & 13.21 \\
\hline
\end{tabular}

Significant differences $(p<0.05)$ are highlighted in bold. Consecutive stages and other pairwise comparisons do not exhibit any significant differences.

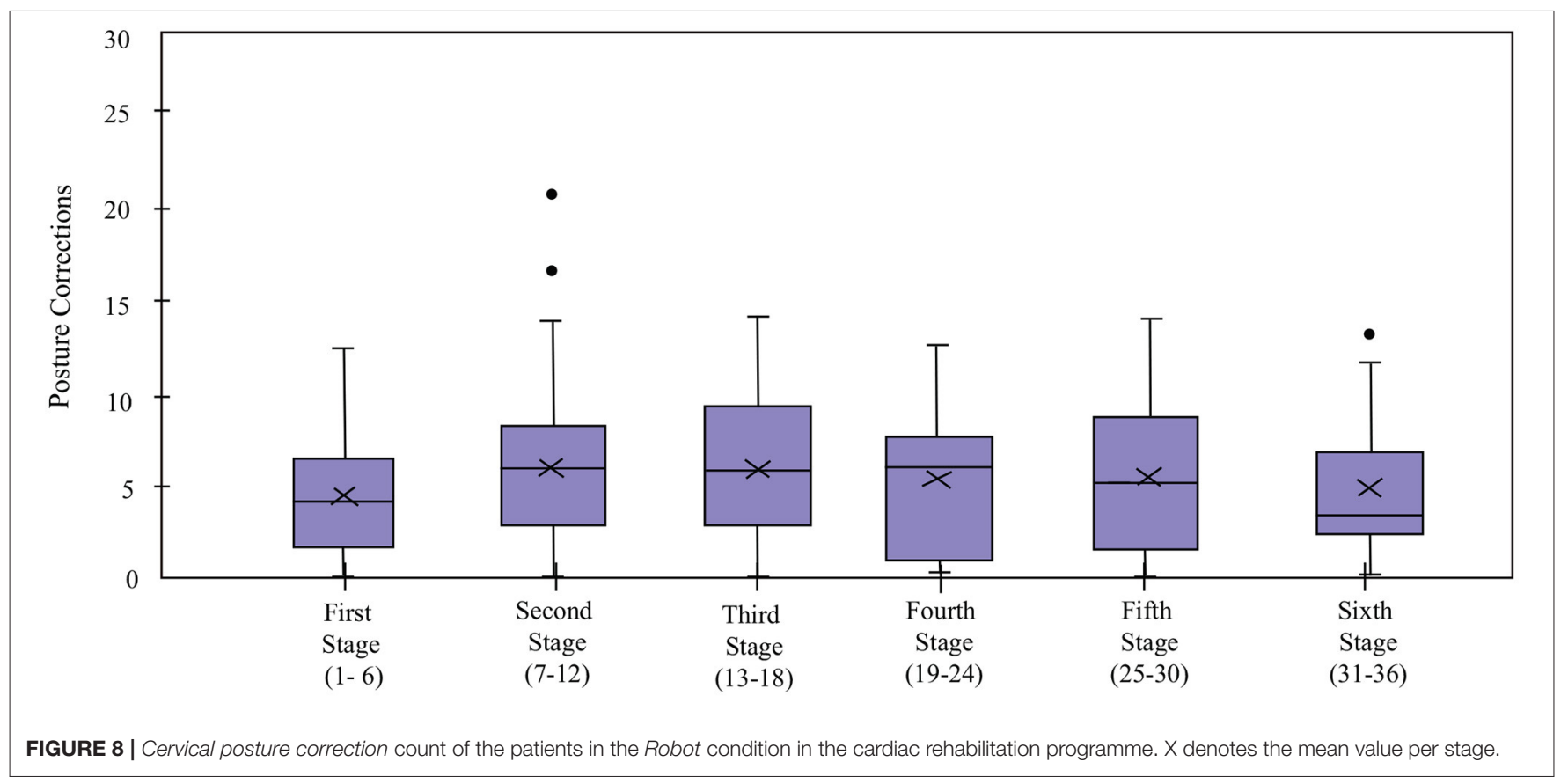

feedback and the motivation given to the patients to improve their exercise quality.

Cardiac rehabilitation aims to accelerate recovery and reduce the risk of suffering recurrent events through structured exercises that progressively increase in intensity throughout the programme. The recovery heart rate $(R H R)$ is the primary physiological parameter that determines the CR progress. Our results show that the patients assisted by the robot achieved a significantly better recovery overall, obtained a higher increase in $R H R$, exceeded the healthy threshold more frequently, and had a more rapid improvement compared to their initial status, thus, a better cardiovascular capacity functioning. These important clinical results strongly support the use of a socially assistive robot for CR programmes. On the other hand, the results in the Control group may be due to the behavior of the RHR of two patients, which show a decrease over the rehabilitation procedure.

There were no significant differences in the perceived level of exertion (Borg Scale) between the conditions, which was to be expected, as the physical activity intensity parameters (i.e., Treadmill's inclination and Gait Speed) for both groups 


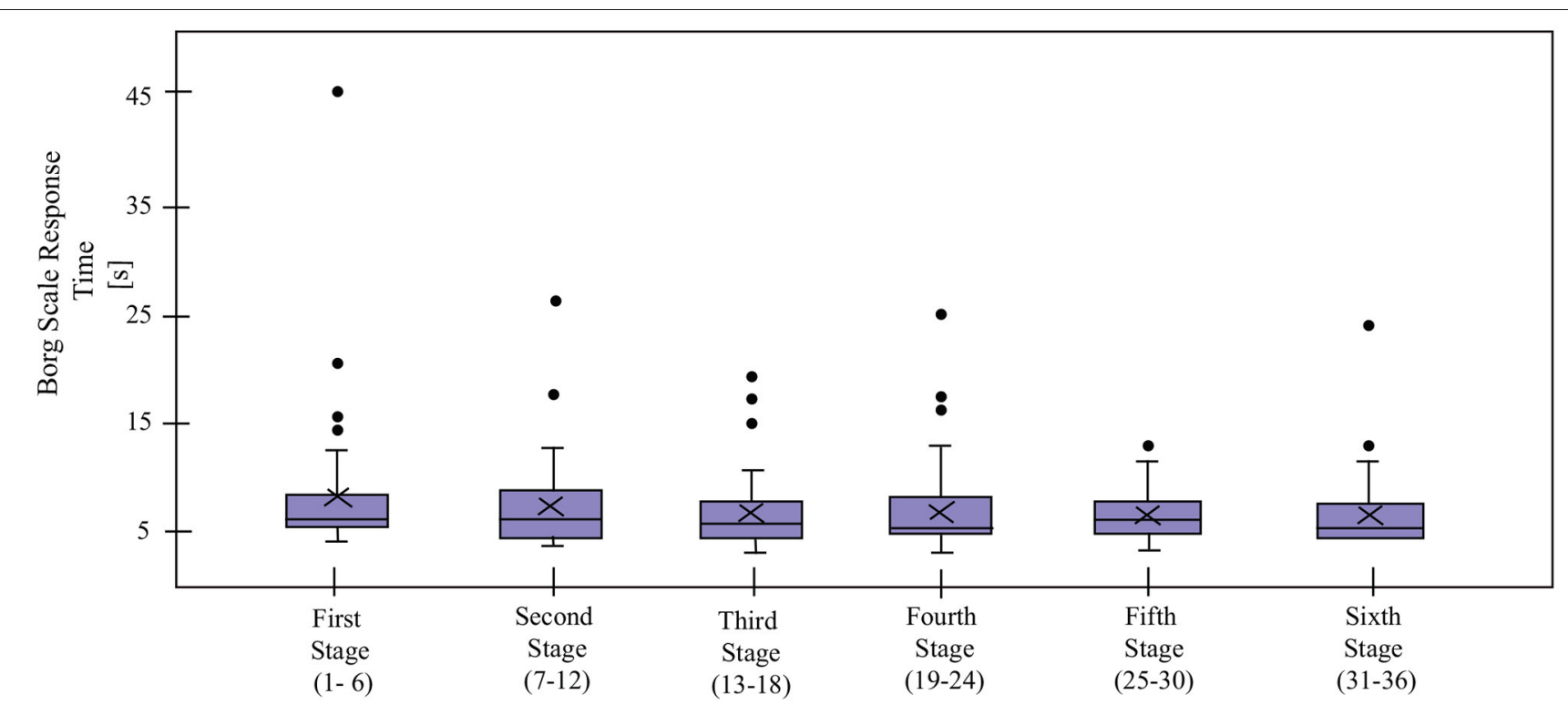

FIGURE 9 | Borg Scale Response Time for the patients in the Robot condition in the cardiac rehabilitation programme. X denotes the mean value per stage.

were prescribed homogeneously (i.e., no significant differences between the conditions). In contrast, the results exhibit significant differences between stages, demonstrating that the perceived exertion level changes across the duration of the CR programme and depends on the physical activity intensity parameters. For instance, if the Treadmill's inclination increases the patient can perceive a higher level of exertion. In particular, for the Control condition, the changes were more evident between the initial stage and the later stages (4 and 5) than the Robot condition where the comparison between stages did not present differences. This result could suggest that the patients in the Robot condition managed to maintain the perceived exertion despite the increase in physical activity, which further support the improvement on their cardiovascular functioning.

Due to the progressively increasing physical activity intensity in the sessions, the training heart rate (THR) varies throughout the CR programme. While this change is significant from the initial stage for the patients that were assisted by a robot, no significant differences were found for the Control condition. This result correlates with the behavior of the RHR, showing that the patients assisted by the robot perform better rehabilitation. These results indicate that the robot has substantial positive effects on the physiological progress of the patients. The underlying reason could be the robot's positive influence on the patients' willingness to achieve the physical activity goals in each session. Furthermore, this outcome can suggest Furthermore, this outcome can suggest that the robot has an influence in the patient's intrinsic motivation, not only through its motivational aspects, but also because of the perceived physiological benefits, which may have improved the patient engagement in the exercise and the CR sessions, because it is personally rewarding.
The monitoring of the physiological data is a critical feature in $\mathrm{CR}$ and allow the medical staff to have a better knowledge about the patient's progress. In comparison to the other studies mentioned in the related work section (Kang et al., 2005; Matarić et al., 2007b), this work presents a deeper analysis of the physiological data of patients assisted by a social robot in cardiac rehabilitation.

The continuous monitoring of the Cervical Posture by the robot enabled corrective feedback (Figure 8), thereby, reducing the risk of dizziness and falls. The results suggest that the patients managed to maintain their cervical posture throughout the programme.

The Borg Scale Response Time (Figure 9) suggests that despite not having prior experience with robots and coming from differing educational backgrounds, all the patients quickly adapted to the technology, and maintained their interaction with the robot throughout the long-term rehabilitation programme. In addition, the experimenters observed that at the beginning of the rehabilitation, the patients take more time to understand the voice and the indications of the robot. Over time, this interaction becomes more fluid due to the experience they acquire. This result shows the importance of the learning curve and how they successfully overcome it. Suggesting an adaptation toward the technology allows long-term interactions and a positive acceptance (Leite et al., 2013). Continuous monitoring of the heart rate enabled providing immediate feedback to alert the medical staff in case of emergencies, which enabled rapid intervention (e.g., through decreasing the physical activity intensity parameters) from them. Hence, despite the fact that the robot malfunctioned and gave an excessive number of alerts on some occasions, this alert proved to be valuable to the medical staff (Irfan et al., 2020). 


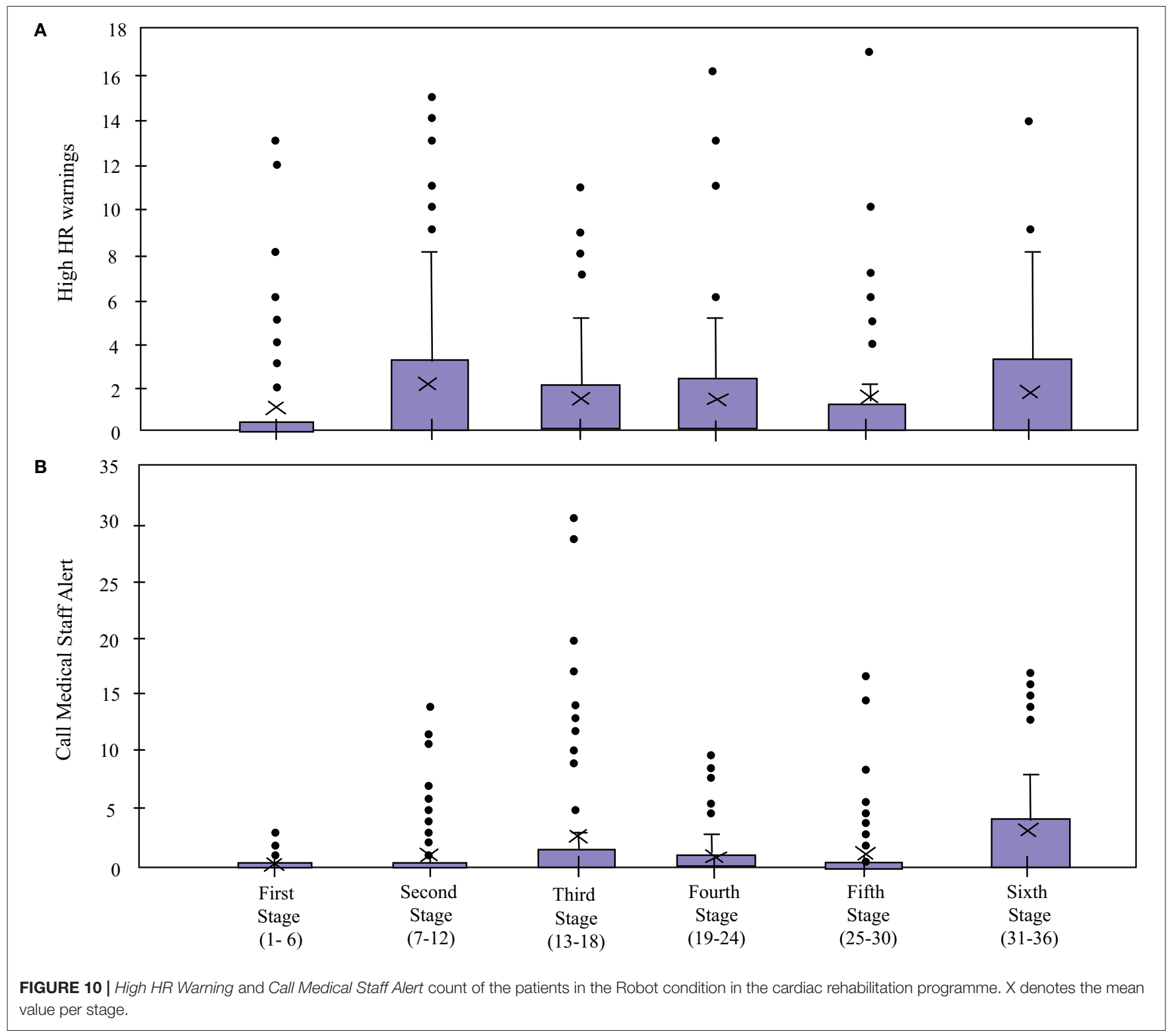

\subsection{Potential for Robots in Rehabilitation}

This paper presents a real-world scenario for long-term application of a socially assistive robot in cardiac rehabilitation. As stated in section 2, few published studies include a social robot in real-world long-term rehabilitation scenarios and fewer in cardiac rehabilitation. Therefore, this work is a starting point for these applications and the opportunity to improve the quality of life of the patients that suffer from cardiovascular diseases and require cardiac rehabilitation.

While previous work made clear how physically interactive robots could support rehabilitation programmes, our results show that purely socially assistive robots can have a positive impact on CR both on the patients and on the medical staff. First of all, SAR can improve adherence, which prevents up to $50 \%$ of the patients worldwide from completing their CR programme (Carlson et al., 2000; Scane et al., 2012), and increase motivation to complete the $\mathrm{CR}$ programme faster. Second, we have indications that the social robot could improve cardiovascular functioning, indicating an improvement in the success of the programme. Finally, both the patients and the medical staff reported that they appreciated the robot, acknowledged that it improves the motivation, engagement and trust in the CR programme, and recommended its use for future patients and healthcare staff. The patients perceived the robot as a coaching partner, who help them to carry on their exercise routine throughout the $\mathrm{CR}$ programme. In addition, they felt more secure due to the continuous monitoring aspects based on the sensor interface, which allowed them to obtain realtime feedback. As shown in the results, these measurements can decrease risk factors in developing physical activity by giving 


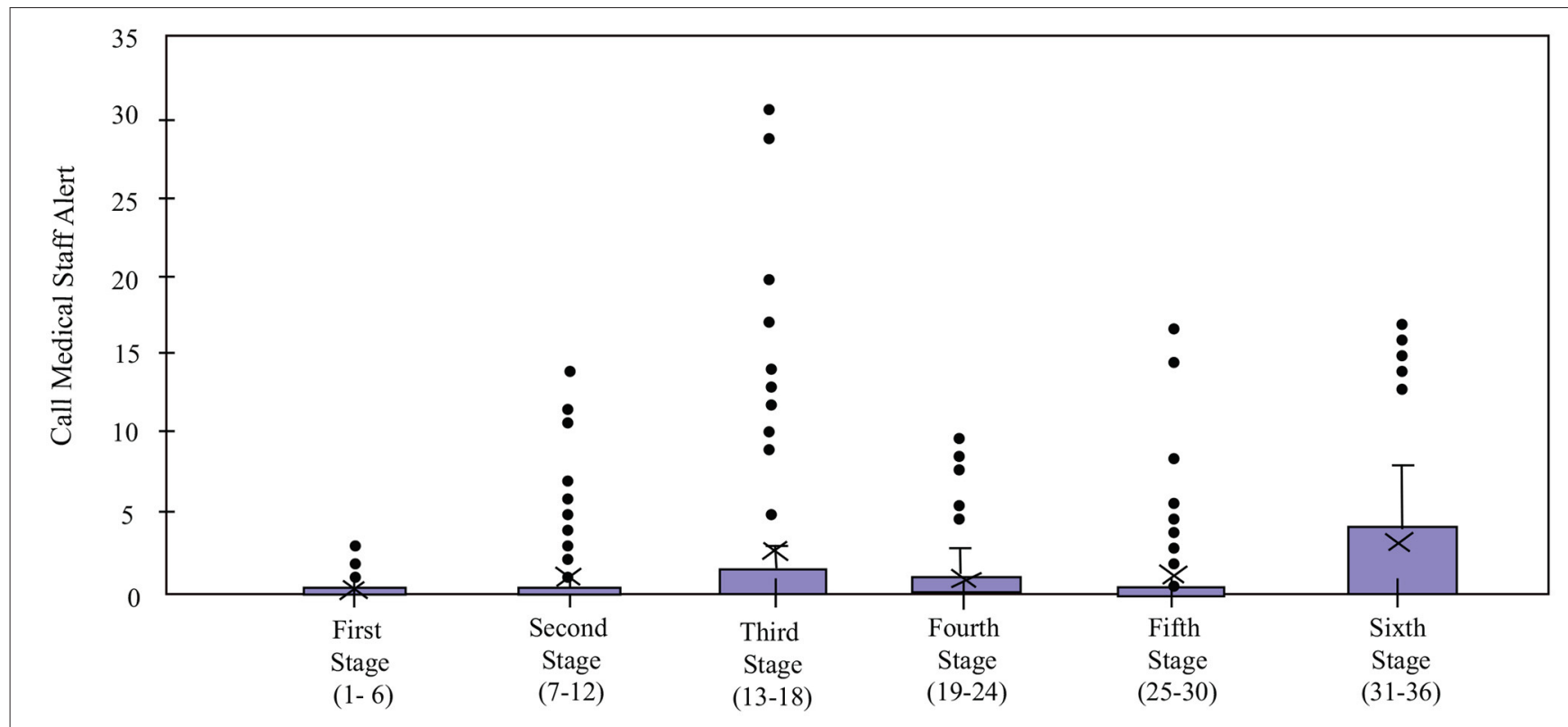

FIGURE 11 | Call Medical Staff Alert count of the patients in the Robot condition in the cardiac rehabilitation programme. X denotes the mean value per stage.

feedback to the patient and the healthcare staff. This interface could also assess the impact of new strategies with social robots in the patients' physiological state. The patient's adaptation to the robot using interaction based on a touchscreen interface, as shown by the Borg scale response time could be also used in other applications where there is a noisy environment in which the patient may have a difficulty understanding the robot. Important to note is that the medical staff also reported finding the continuous monitoring and immediate feedback of the robot valuable, since the added assurance of the robot's monitoring allows them to focus on the person-to-person interaction with the patient and facilitates rapid intervention in case of emergencies.

\subsection{Experimental Limitations and Future Work}

Nevertheless, our study did have a number of limitations. First, due to the random assignation of patients in the conditions and the relatively limited number of patients, our evaluation had some imbalance between the populations (e.g., unequal gender, obesity, differing education levels). This could be addressed in the future by using larger populations or crossover designs, for example by forming pairs of similar participants and dividing them into different conditions. However, as we recruited participants progressively throughout the study, this method was complex to apply to our study. A second limitation is that our robot's behaviors were identical for each participant, which led some participants to report that they would like the robot to be more personalized. We are currently exploring personalization strategies (Irfan et al., 2020) (e.g., recognizing patients, using their name, and referring to progress in the previous sessions) to address these concerns and improve the adaptation to the robot without the perception of repetitiveness or boredom. Moreover, the patients in the Control condition did not receive motivational or physiological verbal feedback to emulate the conventional $\mathrm{CR}$ sessions. While most of the physiological parameters were displayed on the tablet interface for the convenience of the medical staff, the lack of any feedback for cervical posture may have affected the results. The robustness of the interface was another limitation presented during the study, as the heart rate sensor was disconnected on some occasions and there was a loss in data. This limitation is being addressed with the development of a new software architecture based on modules to avoid the problems in the acquisition of the sensors. Finally, limitations caused by the malfunctioning of the fully autonomous robot platform and sensors were also present, such as the high number of alerts triggered for the High HR values and some failures on the medical sensors because they were constantly in use for long periods. This last limitation in the sensors was addressed with the acquisition of a new set of sensors.

Other future work could include the interaction with the social robot in different stages of the CR, like warming up and cool down. This interaction could also continue in the patients' homes through virtual agents that could reinforce the healthy habits taught in the CR programme. Concerning the robot system, it could be possible to test other robot platforms like Pepper that could move around in the rehabilitation center and do followups in several patients simultaneously. Finally, integrating other relevant patient measurements would help better understand the patients' performance and guide their CR progress adequately. 
In this sense, approaches focused on fatigue estimation extracted from cameras and wearable sensors (Pinto et al., 2020) could promote motivation when there are low levels of fatigue, and warning when there are overtraining situations.

\section{CONCLUSIONS}

This paper presented the integration of a social robot into the outpatient phase (II) of a cardiac rehabilitation programme. The primary role of the social robot was to assist patients throughout the sessions using various kinds of feedback and interactions. To assess the effect of the robot on the rehabilitation outcomes, two conditions were designed: (i) a Control condition, where the patient is enrolled in a conventional CR programme, and (ii) a Robot condition, where the patient is assisted and supported by the social robot. A total of 30 patients (15 patients in the Control condition and 15 in the Robot condition) were included in the study.

However, only 20 patients completed the outpatient phase (9 Control condition patients and 11 Robot condition), due to dropouts and other external factors. Four main positive conclusions can be drawn from this study: (i) the dropout rate was lower in the Robot condition and the patients completed 36 sessions of the programme in a shorter time, (ii) physiological outcomes, as measured by the recovery heart rate, were significantly better in the Robot group, and the patients more frequently reached an optimal exercise regime, which indicate a greater improvement in their cardiovascular functioning and recovery, (iii) the patients maintained their interaction with the robot throughout the long-term CR programme, (iv) the clinicians and the patients found the robot valuable for improving perceived safety, patient motivation, and adherence, and recommended its use for future patients and healthcare staff. These results showed the potential of a socially assistive robot in cardiac rehabilitation as a tool to improve the conventional sessions.

\section{DATA AVAILABILITY STATEMENT}

The raw data supporting the conclusions of this article will not be made available by the authors as it contains reserved clinical information. Requests to access the datasets should be directed to marcela.munera@escuelaing.edu.co.

\section{REFERENCES}

Aamot, I. L., Forbord, S. H., Karlsen, T., and Støylen, A. (2014). Does rating of perceived exertion result in target exercise intensity during interval training in cardiac rehabilitation? A study of the Borg scale versus a heart rate monitor. J. Sci. Med. Sport 17, 541-545. doi: 10.1016/j.jsams.2013.07.019

Aguirre, A., Sierra, S. D., Múnera, M., and Cifuentes, C. A. (2020). Online system for gait parameters estimation using a lrf sensor for assistive devices. IEEE Sens. J. doi: 10.1109/JSEN.2020.3028279

Altenhoener, T., Leppin, A., Grande, G., and Romppel, M. (2005). Social inequality in patients? physical and psychological state and participation in rehabilitation after myocardial infarction in Germany. Int. J. Rehabil. Res. 28, 251-257. doi: 10.1097/00004356-200509000-00008

\section{ETHICS STATEMENT}

The studies involving human participants were reviewed and approved by Fundación Cardioinfantil-Instituto de Cardiología Ethics Committee. The patients/participants provided their written informed consent to participate in this study. Written informed consent was obtained from the individual(s) for the publication of any potentially identifiable images or data included in this article.

\section{AUTHOR CONTRIBUTIONS}

NC performed the clinical assessment and data processing. $\mathrm{NC}$ and BI performed the statistical analysis and led the manuscript writing. BI and ES contributed with the development of the patient-robot interface for cardiac rehabilitation. MR-R and LG managed the experimental protocol and participated in the clinicians questionnaire development. MM developed the experimental protocol. CC proposed and supervised the structure of the paper. MM, CC, ES, and TB were involved in the revisions and corrections of the manuscript. All authors contributed to the article and approved the submitted version.

\section{FUNDING}

This work was supported in part by the Royal Academy of Engineering IAPP project Human-Robot Interaction Strategies for Rehabilitation based on Socially Assistive Robotics (grant IAPP \1516 \137), Minciencias Colombia (grant 813-2017), the EU H2020 Marie Skłodowska-Curie Actions ITN project APRIL (grant 674868), the EU FP7 project DREAM (grant 611391), and the Flemish Government (AI Research Program).

\section{ACKNOWLEDGMENTS}

This work was carried out in collaboration with the Fundación Cardioinfantil-Instituto de Cardiología, where the experiments and protocols were performed. The authors would like to thank the patients and the clinicians for their participation, time and efforts. 
Bickmore, T. W., and Picard, R. W. (2005). Establishing and maintaining longterm human-computer relationships. ACM Trans. Comput. Hum. Interact. 12, 293-327. doi: 10.1145/1067860.1067867

Borg, G. (1998). Borg's Perceived Exertion and Pain Scales. Champaign, IL: Human Kinetics.

Carlson, J., Johnson, J., Franklin, B., and VanderLaan, R. (2000). Program participation, exercise adherence, cardiovascular outcomes, and program cost of traditional versus modified cardiac rehabilitation. Am. J. Cardiol. 86, 17-23. doi: 10.1016/S0002-9149(00)00822-5

Carnethon, M., Sternfeld, B., Liu, K., Jcob, D., Schreiner, P., Williams, D., et al. (2015). Correlates of heart rate recovery over 20 years in a population sample. J. Investig. Dermatol. 135, 612-615. doi: 10.1249/MSS.0b013e31822cb190

Carrillo, F. M., Butchart, J., Kruse, N., Scheinberg, A., Wise, L., and McCarthy, C. (2018). "Physiotherapists' acceptance of a socially assistive robot in ongoing clinical deployment," in 2018 27th IEEE International Symposium on Robot and Human Interactive Communication (RO-MAN) (Nanjing), 850-855. doi: 10.1109/ROMAN.2018.8525508

Casas, J., Céspedes, N., Cifuentes, C., Gutierrez, L. F., Rincón-Roncancio, M., and Múnera, M. (2019). Expectation vs. reality: Attitudes towards a socially assistive robot in cardiac rehabilitation. Appl. Sci. 9:4651. doi: 10.3390/app9214651

Casas, J., Gomez, N. C., Senft, E., Irfan, B., Gutiérrez, L. F., Rincón, M., et al. (2018c). "Architecture for a social assistive robot in cardiac rehabilitation," in 2018 IEEE 2nd Colombian Conference on Robotics and Automation (CCRA) (Barranquilla), 1-6. doi: 10.1109/CCRA.2018.8588133

Casas, J., Irfan, B., Senft, E., Gutiérrez, L., Rincon-Roncancio, M., Munera, M., et al. (2018a). "Social assistive robot for cardiac rehabilitation: a pilot study with patients with angioplasty," in Companion of the 2018 ACM/IEEE International Conference on Human-Robot Interaction (Chicago, IL: ACM), 79-80. doi: $10.1145 / 3173386.3177052$

Casas, J., Irfan, B., Senft, E., Gutierrez, L., Rincon-Roncancio, M., Munera, M., et al. (2018b). "Towards a sar system for personalized cardiac rehabilitation: a patient with PCI," in 2018 ACM/IEEE International Conference on Human-Robot Interaction Personal Robots for Exercising and Coaching Workshop (Chicago, IL: ACM).

Casas, J., Senft, E., Gutierrez, L. F., Rincon-Rocancio, M., Munera, M., Belpaeme, T., et al. (2020). Social assistive robots: assessing the impact of a training assistant robot in cardiac rehabilitation. Int. J. Soc. Robot. 13, 1-15. doi: $10.1007 /$ s12369-020-00708-y

Clark, A. M., Hartling, L., Vandermeer, B., and McAlister, F. A. (2005). Meta-analysis: secondary prevention programs for patients with coronary artery disease. Ann. Intern. Med. 143, 659-672. doi: 10.7326/0003-4819-143-9-200511010-00010

Cohen, J. (1988). Statistical Power Analysis for the Behavioral Sciences. New York, NY: Routledge Academic.

Cooper, A. F., Weinman, J., Hankins, M., Jackson, G., and Horne, R. (2007). Assessing patients' beliefs about cardiac rehabilitation as a basis for predicting attendance after acute myocardial infarction. Heart 93, 53-58. doi: 10.1136/hrt.2005.081299

Eriksson, J., Matarić, M. J., and Winstein, C. J. (2005). "Hands-off assistive robotics for post-stroke arm rehabilitation," in 9th International Conference on Rehabilitation Robotics, 2005, ICORR 2005 (Chicago, IL), 21-24. doi: 10.1109/ICORR.2005.1501042

Fasola, J., and Matarić, M. J. (2010). "Robot exercise instructor: a socially assistive robot system to monitor and encourage physical exercise for the elderly," in Proceedings - IEEE International Workshop on Robot and Human Interactive Communication (Viareggio), 416-421. doi: 10.1109/ROMAN.2010.5598658

Fasola, J., and Mataric, M. J. (2012). Using socially assistive human-robot interaction to motivate physical exercise for older adults. Proc. IEEE 100, 2512-2526. doi: 10.1109/JPROC.2012.2200539

Fasola, J., and Matarić, M. J. (2013). A socially assistive robot exercise coach for the elderly. J. Hum. Robot Interact. 2, 3-32. doi: 10.5898/JHRI.2.2.Fasola

Feil-Seifer, D., and Matarić, M. J. (2005). "Defining socially assistive robotics," in 9th International Conference on Rehabilitation Robotics (ICORR) (Chicago, IL), 465-468. doi: 10.1109/ICORR.2005.1501143

Gadde, P., Kharrazi, H., Patel, H., and MacDorman, K. F. (2011). Toward monitoring and increasing exercise adherence in older adults by robotic intervention: a proof of concept study. J. Robot. 2011, 1-11. doi: $10.1155 / 2011 / 438514$
Galve, E., Alegria, E., Cordero, A., Facila, L., de Bobadilla, J. F., LluisGanella, C., et al. (2014). Temas de actualidad en cardiologia: riesgo vascular y rehabilitacion cardiaca. Revista Espanola de Cardiologia 67, 203-210. doi: 10.1016/j.recesp.2013.09.021

Giuliano, C., Parmenter, B. J., Baker, M., Mitchell, B. L., Williams, A. D., Lyndon, K., et al. (2017). Cardiac rehabilitation for patients with coronary artery disease: a practical guide to enhance patient outcomes through continuity of care. Clin. Med. Insights Cardiol. 11, 1-7. doi: 10.1177/1179546817710028

Glass, G. V., McGaw, B., and Smith, M. L. (1981). Meta-Analysis Insocial Research. Newbury Park, CA: Sage.

Gockley, R., Bruce, A., Forlizzi, J., Michalowski, M., Mundell, A., Rosenthal, S., et al. (2005). "Designing robots for long-term social interaction," in 2005 IEEE/RSI International Conference on Intelligent Robots and Systems (Edmonton, AB), 1338-1343. doi: 10.1109/IROS.2005.1545303

Gockley, R., and Mataric, M. J. (2006). "Encouraging physical therapy compliance with a hands-off mobile robot," in Proceedings of the 1st ACM SIGCHI/SIGART Conference on Human-Robot Interaction, HRI?06 (New York, NY: Association for Computing Machinery), 150-155. doi: 10.1145/1121241.1121268

Hammill, B. G., Curtis, L. H., Schulman, K. A., and Whellan, D. J. (2010). Relationship between cardiac rehabilitation and long-term risks of death and myocardial infarction among elderly medicare beneficiaries. Circulation 121, 63-70. doi: 10.1161/CIRCULATIONAHA.109.876383

Irfan, B., Céspedes Gomez, N., Casas, J., Senft, E., Gutiérrez, L. F., RinconRoncancio, M., et al. (2020). "Using a personalised socially assistive robot for cardiac rehabilitation: a long-term case study," in 29th IEEE International Symposium on Robot and Human Interactive Communication (RO-MAN) (IEEE), 124-130. doi: 10.1109/RO-MAN47096.2020.9223491

James, G. S. (1951). The comparison of several groups of observations when the ratios of the population variances are unknown. Biometrika 38, 324-329. doi: 10.1093/biomet/38.3-4.324

Johansen, S. (1980). The welch-james approximation to the distribution of the residual sum of squares in a weighted linear regression. Biometrika 67, 85-92. doi: 10.1093/biomet/67.1.85

Jolly, K., Taylor, R., Lip, G., Greenfield, S., Raftery, J., Mant, J., et al. (2007). The Birmingham rehabilitation uptake maximisation study (brum). Homebased compared with hospital-based cardiac rehabilitation in a multi-ethnic population: cost-effectiveness and patient adherence. Health Technol. Assess. 11,1-118. doi: 10.3310/hta11350

Kang, K. I., Freedman, S., Mataric, M. J., Cunningham, M. J., and Lopez, B. (2005). "A hands-off physical therapy assistance robot for cardiac patients," in 9th International Conference on Rehabilitation Robotics, 2005 (ICORR 2005) (Chicago, IL), 337-340. doi: 10.1109/ICORR.2005.1501114

Keselman, H. J., Wilcox, R. R., and Lix, L. M. (2003). A generally robust approach to hypothesis testing in independent and correlated groups designs. Psychophysiology 40, 586-596. doi: 10.1111/1469-8986.00060

Kidd, C. D., and Breazeal, C. (2007). "A robotic weight loss coach," in Proceedings of the 22nd National Conference on Artificial Intelligence (Vancouver, BC: AAAI Press), 1985-1986.

Kraus, W., and Keteyian, S. (2007). Cardiac Rehabilitation. Totowa, NJ: Humana Press. doi: 10.1007/978-1-59745-452-0

Lane, G. W., Noronha, D., Rivera, A., Craig, K., Yee, C., Mills, B., et al. (2016). Effectiveness of a social robot, "Paro," in a VA long-term care setting. Psychol. Serv. 13, 292-299. doi: 10.1037/ser0000080

Langer, A., Feingold-Polak, R., Mueller, O., Kellmeyer, P., and Levy-Tzedek, S. (2019). Trust in socially assistive robots: considerations for use in rehabilitation. Neurosci. Biobehav. Rev. 104, 231-239. doi: 10.1016/j.neubiorev.2019.07.014

Lara, J. S., Casas, J., Aguirre, A., Munera, M., Rincon-Roncancio, M., Irfan, B., et al. (2017). "Human-robot sensor interface for cardiac rehabilitation," in 2017 International Conference on Rehabilitation Robotics (ICORR) (London), 1013-1018. doi: 10.1109/ICORR.2017.8009382

Lawler, P. R., Filion, K. B., and Eisenberg, M. J. (2011). Efficacy of exercise-based cardiac rehabilitation post-myocardial infarction: a systematic review and meta-analysis of randomized controlled trials. Am. Heart J. 162, 571.e2-584.e2. doi: 10.1016/j.ahj.2011.07.017

Leite, I., Martinho, C., and Paiva, A. (2013). Social robots for long-term interaction: a survey. Int. J. Soc. Robot. 5, 291-308. doi: 10.1007/s12369-013-0178-y

Lemaignan, S., Garcia, F., Jacq, A., and Dillenbourg, P. (2016). "From real-time attention assessment to "with-me-ness" in human-robot interaction," in 2016 
11th ACM/IEEE International Conference on Human-Robot Interaction (HRI) (Christchurch: IEEE), 157-164. doi: 10.1109/HRI.2016.7451747

Li, J. (2015). The benefit of being physically present. Int. J. Hum. Comput. Stud. 77, 23-37. doi: 10.1016/j.ijhcs.2015.01.001

Maclean, N., and Pound, P. (2000). A critical review of the concept of patient motivation in the literature on physical rehabilitation. Soc. Sci. Med. 50, 495-506. doi: 10.1016/S0277-9536(99)00334-2

Martin, C. M., and McConahay, D. R. (1972). Maximal treadmill exercise electrocardiography. Correlations with coronary arteriography and cardiac hemodynamics. Circulation 46, 956-962. doi: 10.1161/01.CIR.46.5.956

Matarić, M., Eriksson, J., Feil-Seifer, D., and Winstein, C. J. (2007a). Socially assistive robotics for post-stroke rehabilitation. J. Neuroeng. Rehabil. 4, 1-9. doi: 10.1186/1743-0003-4-5

Matarić, M., and Scassellati, B. (2016). "Socially assistive robotics," in Springer Handbook of Robotics, eds B. Siciliano and O. Khatib (Cham: Springer), 19731993. doi: 10.1007/978-3-319-32552-1_73

Matarić, M., Tapus, A., and Feil-Seifer, D. (2007b). "Personalized socially assistive robotics," in Workshop on Intelligent Systems for Assisted Cognition Rochester, NY.

McKee, G., Biddle, M., Donnell, S. O., Mooney, M., Brien, F. O., and Moser, D. K. (2014). Cardiac rehabilitation after myocardial infarction: What influences patients? intentions to attend? Eur. J. Cardiovasc. Nurs. 13, 329-337. doi: $10.1177 / 1474515113496686$

Oldridge, N. B., Guyatt, G. H., Fischer, M. E., and Rimm, A. A. (1988). Cardiac rehabilitation after myocardial infarction: combined experience of randomized clinical trials. JAMA 260, 945-950. doi: 10.1001/jama.260.7.945

Piepoli, M. F., Corre, U., Benzer, W., Bjarnason-Wehrens, B., Dendale, P., Gaita, D., et al. (2010). Secondary prevention through cardiac rehabilitation: from knowledge to implementation. A position paper from the cardiac rehabilitation section of the European Association of Cardiovascular Prevention and Rehabilitation. Eur. J. Cardiovasc. Prev. Rehabil. 17, 1-17. doi: 10.1097/HJR.0b013e3283313592

Pinto, M. J., Aguirre, A., Cifuentes, C. A., and Munera, M. (2020). "Wearable sensors for monitoring exercise and fatigue estimation in rehabilitation," in Internet of Medical Things: Paradigm of Wearable Devices, eds M. Cardona, V. K. Solanki, and C. E. García Cena (Boca Raton, FL: Taylor \& Francis Group).

Riek, L. D. (2017). Healthcare robotics. Commun. ACM 60, 68-78. doi: $10.1145 / 3127874$

Ruano-Ravina, A., Pena-Gil, C., Abu-Assi, E., Raposeiras, S., van 't Hof, A., Meindersma, E., et al. (2016). Participation and adherence to cardiac rehabilitation programs. A systematic review. Int. J. Cardiol. 223, 436-443. doi: 10.1016/j.ijcard.2016.08.120

Šabanović, S., Bennett, C. C., Chang, W., and Huber, L. (2013). "Paro robot affects diverse interaction modalities in group sensory therapy for older adults with dementia," in 2013 IEEE 13th International Conference on Rehabilitation Robotics (ICORR) (Seattle, WA: IEEE), 1-6. doi: 10.1109/ICORR.2013.6650427

Scane, K., Alter, D., Oh, P., and Brooks, D. (2012). Adherence to a cardiac rehabilitation home program model of care: a comparison to a well-established traditional on-site supervised program. Appl. Physiol. Nutr. Metab. 37, 206-213. doi: 10.1139/h11-151

Schafer, R., and Anderson, J. (1987). Clinical Biomechanics. Baltimore, MD: Williams \& Wilkins.

Shahsavari, H., Shahriari, M., and Alimohammadi, N. (2012). Motivational factors of adherence to cardiac rehabilitation. Iran. J. Nurs. Midwif. Res. 17, 318-324.

She, J., Nakamura, H., Makino, K., Ohyama, Y., and Hashimoto, H. (2014). Selection of suitable maximum-heart-rate formulas for use with Karvonen formula to calculate exercise intensity. Int. J. Automat. Comput. 12, 62-69. doi: $10.1007 /$ s11633-014-0824-3

Siegert, R. J., and Taylor, W. J. (2004). Theoretical aspects of goalsetting and motivation in rehabilitation. Disabil. Rehabil. 26, 1-8. doi: 10.1080/09638280410001644932

Suaya, J., Stason, W., Ades, P., Normand, S., and Shepard, D. (2009). Cardiac rehabilitation and survival in older coronary patients. J. Am. Coll. Cardiol. 54, 25-33. doi: 10.1016/j.jacc.2009.01.078

Süssenbach, L., Riether, N., Schneider, S., Berger, I., Kummert, F., Lutkebohle, I., et al. (2014). "A robot as fitness companion: towards an interactive action-based motivation model," in The 23rd IEEE International Symposium on Robot and Human Interactive Communication (Edinburgh), 286-293. doi: 10.1109/ROMAN.2014.6926267

Swift-Spong, K., Short, E., Wade, E., and Mataric, M. J. (2015). "Effects of comparative feedback from a socially assistive robot on self-efficacy in poststroke rehabilitation," in 2015 IEEE International Conference on Rehabilitation Robotics (ICORR) (Nanyang), 764-769. doi: 10.1109/ICORR.2015. 7281294

Taylor, R., Brown, A., and Ebrahim, S. A. (2012). Exercise-based cardiac rehabilitation in patients with coronary heart disease: meta-analysis outcomes revisited. Future Cardiol. 8, 729-751. doi: 10.2217/fca.12.34

Taylor, R. S., Brown, A., Ebrahim, S., Jolliffe, J., Noorani, H., Rees, K., et al. (2004). Exercise-based rehabilitation for patients with coronary heart disease: systematic review and meta-analysis of randomized controlled trials. Am. J. Med. 116, 682-692. doi: 10.1016/j.amjmed.2004.01.009

Thomas, R. J., King, M., Lui, K., Oldridge, N., Pina, I. L., Spertus, J., et al. (2007). AACVPR/ACC/AHA 2007 performance measures on cardiac rehabilitation for referral to and delivery of cardiac rehabilitation/secondary prevention services. J. Cardiopulm. Rehabil. Prev. 27, 260-290. doi: 10.1097/01.HCR.0000291295.24776.7b

Thompson, D. (2002). Stride Analysis. Available online at: https://ouhsc.edu/ bserdac/dthompso/web/gait/knmatics/stride.htm (accessed June 16, 2020).

Turk-Adawi, K., Supervia, M., Lopez-Jimenez, F., Pesah, E., Ding, R., Britto, R. R., et al. (2019). Cardiac rehabilitation availability and density around the globe. eClinicalmedicine 13, 31-45. doi: 10.1016/j.eclinm.2019.06.007

Turk-Adawi, K. I., Oldridge, N. B., Tarima, S. S., Stason, W. B., and Shepard, D. S. (2013). Cardiac rehabilitation patient and organizational factors: what keeps patients in programs? J. Am. Heart Assoc. 2:e000418. doi: 10.1161/JAHA.113.000418

Vasco, V., Willems, C., Chevalier, P., De Tommaso, D., Gower, V., Gramatica, F., et al. (2019). "Train with me: a study comparing a socially assistive robot and a virtual agent for a rehabilitation task," in International Conference on Social Robotics (ICSR 2019) (Madrid: Springer). doi: 10.1007/978-3-030-358 88-4 42

Venkatesh, V., Morris, M. G., Davis, G. B., and Davis, F. D. (2003). User acceptance of information technology: toward a unified view. MIS Q. 27, 425-478. doi: 10.2307/30036540

Villacorta, P. J. (2017). The welchADF package for robust hypothesis testing in unbalanced multivariate mixed models with heteroscedastic and non-normal data. R J. 9, 309-328. doi: 10.32614/RJ-2017-049

Welch, B. L. (1938). The significance of the difference between two means when the population variances are unequal. Biometrika 29, 350-362. doi: 10.1093/biomet/29.3-4.350

Welch, B. L. (1951). On the comparison of several mean values: an alternative approach. Biometrika 38, 330-336. doi: 10.1093/biomet/38.3-4.330

Winkle, K., Caleb-Solly, P., Turton, A., and Bremner, P. (2018). "Social robots for engagement in rehabilitative therapies: design implications from a study with therapists," in Proceedings of the 2018 ACM/IEEE International Conference on Human-Robot Interaction, HRI?18 (New York, NY: Association for Computing Machinery), 289-297. doi: 10.1145/3171221.3171273

World Health Organization (2011). World Report on Disability, Vol. 91. Geneva: The World Bank.

Conflict of Interest: The authors declare that the research was conducted in the absence of any commercial or financial relationships that could be construed as a potential conflict of interest.

The handling Editor declared a past co-authorship with one of the authors BI.

Copyright (C) 2021 Céspedes, Irfan, Senft, Cifuentes, Gutierrez, Rincon-Roncancio, Belpaeme and Múnera. This is an open-access article distributed under the terms of the Creative Commons Attribution License (CC BY). The use, distribution or reproduction in other forums is permitted, provided the original author(s) and the copyright owner(s) are credited and that the original publication in this journal is cited, in accordance with accepted academic practice. No use, distribution or reproduction is permitted which does not comply with these terms. 